\title{
Dynamic delamination on elastic interface
}

\author{
Tianyu Chen ${ }^{\mathrm{a}}$, Christopher M. Harvey ${ }^{\mathrm{a},}$, Simon Wang, ${ }^{\mathrm{a}, \mathrm{c}}$, Vadim V. Silberschmidt ${ }^{\mathrm{b}}$ \\ ${ }^{a}$ Department of Aeronautical and Automotive Engineering, Loughborough University, \\ Loughborough, Leicestershire LE11 3TU, UK \\ ${ }^{b}$ Wolfson School of Mechanical, Electrical and Manufacturing Engineering, Loughborough University, \\ Loughborough, Leicestershire LE11 3TU, UK
}

${ }^{c}$ School of Mechanical and Equipment Engineering, Hebei University of Engineering, Handan 056038, China

\begin{abstract}
The dynamic energy release rate (ERR) is derived for a delamination on the interface between a partially supported vibrating beam and an elastic foundation, with a time-dependent displacement applied to the beam's free end. The configuration may represent, for example, the dynamic delamination of a laminated composite, or the cracking of a typical adhesively bonded composite joint. The developed theory is completely analytical and applicable to both symmetric double cantilever beams (DCBs) and thin layers on thick substrates. It was discovered that the dispersive propagation of flexural waves should be considered in order to capture contributions to the ERR from higher-order vibration modes. The developed theory is verified using finite-element-method (FEM) simulations and they are found to be in excellent agreement. This work will be useful to characterize the dynamic fracture toughness of layered materials in DCB tests, and to determine the fracture behavior of engineering structures under dynamic loads. Furthermore, the partially supported beam's elastic foundation is relevant for the study of crack process zones, which are usually analyzed using the FEM and the cohesivezone model. The potential applications of this study include determining the dynamic fracture toughness for crack initiation in laminated composite DCBs and adhesively bonded structures.
\end{abstract} Keywords: beam dynamics; delamination; dynamic energy release rate; elastic foundation; vibration

\footnotetext{
* Corresponding Author
}

Email addresses: t. chen3@ lboro.ac.uk (Tianyu Chen),

c.m.harvey@lboro.ac.uk (Christopher M. Harvey),

s.wang@lboro.ac.uk (Simon Wang), 


\begin{tabular}{|c|c|}
\hline \multicolumn{2}{|c|}{ Principal nomenclature } \\
\hline$A$ & Area of cross-section of beam \\
\hline$A_{0}$ & Crack area \\
\hline$b$ & Width of beam \\
\hline$E$ & Young's modulus \\
\hline$f_{\text {dyn }}$ & Dynamic factor due to total dynamic effect \\
\hline$f_{\text {vib }}$ & Dynamic factor due to vibration \\
\hline$F(\Omega)$ & Energy flux \\
\hline$F^{\mathrm{FD}}(x)$ & Shifting function of the foundation-supported beam section \\
\hline$F^{\mathrm{FR}}(x)$ & Shifting function of the free beam section \\
\hline$F_{\text {vib }}(\Omega)$ & Energy flux due to vibration \\
\hline$G$ & Energy release rate \\
\hline$G_{\mathrm{st}}^{\mathrm{K}}$ & ERR component due to kinetic energy of static motion \\
\hline$G_{\mathrm{st}}^{\mathrm{U}}$ & ERR component due to strain energy of static motion \\
\hline$G_{\text {vib }}$ & ERR component due to vibration \\
\hline$h$ & Thickness of beam \\
\hline$I$ & Second moment of area of beam \\
\hline$k$ & Foundation stiffness \\
\hline$K$ & Kinetic energy \\
\hline$S$ & Strain energy stored in the foundation \\
\hline$t$ & Time \\
\hline$T_{i}(t), \dot{T}_{i}(t)$ & Modal displacement and velocity of the $i$ th normal mode \\
\hline$U$ & Strain energy of the whole beam \\
\hline$v$ & Applied constant opening velocity \\
\hline$w^{\mathrm{FD}}(x, t)$ & Total transverse deflection for the beam section on foundation \\
\hline$w_{\mathrm{fv}}^{\mathrm{FD}}(x, t)$ & Deflection for the beam section on foundation due to free vibration \\
\hline$w^{\mathrm{FR}}(x, t)$ & Total transverse deflection for the free beam section \\
\hline$w_{\mathrm{fv}}^{\mathrm{FR}}(x, t)$ & Deflection for the free beam section due to free vibration \\
\hline$W_{\text {ext }}$ & Work done by external force \\
\hline$W_{i}^{\mathrm{FD}}(x)$ & Normal mode for the beam section on foundation \\
\hline$W_{i}^{\mathrm{FR}}(x)$ & Normal mode for the free beam section \\
\hline$\Gamma$ & Energy dissipated to increase crack area \\
\hline$\delta_{i j}$ & Kronecker delta \\
\hline$\Pi$ & Mechanical energy \\
\hline$\rho$ & Density \\
\hline$\omega$ & Angular frequency \\
\hline \multicolumn{2}{|c|}{ Abbreviations } \\
\hline DCB & Double cantilever beam \\
\hline ERR & Energy release rate \\
\hline FEM & Finite-element method \\
\hline VCCT & Virtual crack closure technique \\
\hline
\end{tabular}




\section{Introduction}

The response of structures to time-dependent external excitation can often include significant dynamic effects. It is therefore necessary to consider such effects in the calculation of energy release rate (ERR) when studying the fracture behavior of these structures.

Both quasi-static and dynamic fracture are usually studied for relatively simple structures such as double cantilever beams (DCBs) or thin layers on thick substrates. These structures can capture the relevant physics while not unnecessarily complicating the modelling; the theory developed for them can readily be transferred to more complex structures such as embedded cracks, and axisymmetric plates and shells. They can also represent various real engineering cases such as DCB tests [1] or wedge tests [2] to measure dynamic fracture toughness.

Compared to quasi-static fracture, dynamic fracture has received considerably less research effort. One of the first analytical studies is from Smiley and Pipes [3] for dynamic DCB tests. They used a quasi-static 'crack-opening displacement rate' to determine the kinetic energy and included it in their energy-conservation approach to determine the dynamic ERR. Localized vibration was not considered, and the resulting ERR obtained for a stationary crack was 'smoothed' without any oscillation. Blackman et al. [4] used the same approach to derive the dynamic ERR for steadily propagating cracks in a symmetric DCBs, also without considering localized vibration. Both experiments $[5,6,7]$ and numerical simulations $[8,9,10]$, however, confirm that the dynamic ERR is, in general, oscillatory. To account for vibration, Chen et al. [11] used beam dynamics to model an asymmetric DCB with one very thin layer under general applied displacement. The dynamic ERR was derived by analogy to quasi-static fracture by using the bending moment of a vibrating crack tip. Although the energy-conservation approach was not explicitly used, the calculated dynamic ERR implicitly included kinetic energy as the crack tip bending moment was derived using beam dynamics. Chen at al. [12] subsequently used beam dynamics and the energy-conservation approach to derive an analytical solution for the dynamic ERR of vibrating DCBs under constant opening-rate displacement. The resulting theory was able to capture the first vibration mode's contribution to dynamic ERR but adding higher-order vibration modes caused divergence in the amplitude of ERR oscillation. This divergence was attributed to a limitation of Euler-Bernoulli beams in vibration analysis. Also, in comparison with the FEM simulations, the developed theory was slightly out-of-phase due to the simplified boundary condition, which was a fixed support at the crack tip.

To the best of the authors' knowledge, the theory of dynamic ERR, which includes higherorder vibration, has not been developed before. This is achieved in this work by taking the 
dispersive propagation of flexural waves into account. In addition, for the first time, the dynamic ERR is derived for a stationary delamination (that is, with no propagation) on the interface between a partially supported beam and an elastic foundation. This boundary condition not only substantially corrects the phase difference between the analytical theory [12] and the results of FEM simulations, but also allows non-rigid interfaces to be studied, which is usually done using the FEM and the cohesive-zone model. The elastic foundation can also be considered to represent, for example, a thin adhesive bond, or the interface between the plies of a laminated composite. The developed theory could therefore be used to post-process highloading-rate fracture test data to determine the loading rate-dependent fracture toughness for initiation of stationary cracks in laminated composites or in adhesively bonded structures.

The structure of this paper is as follows: The analytical theory is developed in Section 2, which is then verified against FEM simulations in Section 3. Conclusions are given in Section 4.

\section{Analytical theory}

In this section, the dynamic ERR of the stationary delamination shown in Fig. 1 is derived analytically considering both vibration and wave propagation. A beam in its initial undeformed condition rests on a partial elastic foundation with constant foundation stiffness $k$, with a timedependent displacement $w_{0}(t)$ applied to the midplane of its free end in Fig 1. A constant opening velocity $v$ is selected, that is, $w_{0}(t)=v t$, but the choice is arbitrary, and the theory herein applies under general applied displacement with minor adjustments. The length of the beam section resting on the foundation representing the uncracked region is $L$. The length of the free beam section is $a$, which represents the crack length. The coordinate system is set so that the $x$ axis is to the right with the crack tip at $x=L$, and the $z$ axis is upwards, with the beam's transverse deflection in the $x-z$ plane. The deflection of the foundation-supported beam section is represented by $w^{\mathrm{FD}}(x, t)$, and the deflection of the free beam section is represented by $w^{\mathrm{FR}}(x, t)$. It is assumed that there is no interfacial contact between the free region and the foundation, and that the beam thickness $h$ is small enough compared to $a$ and $L$ for the Euler-Bernoulli beam theory to apply. 


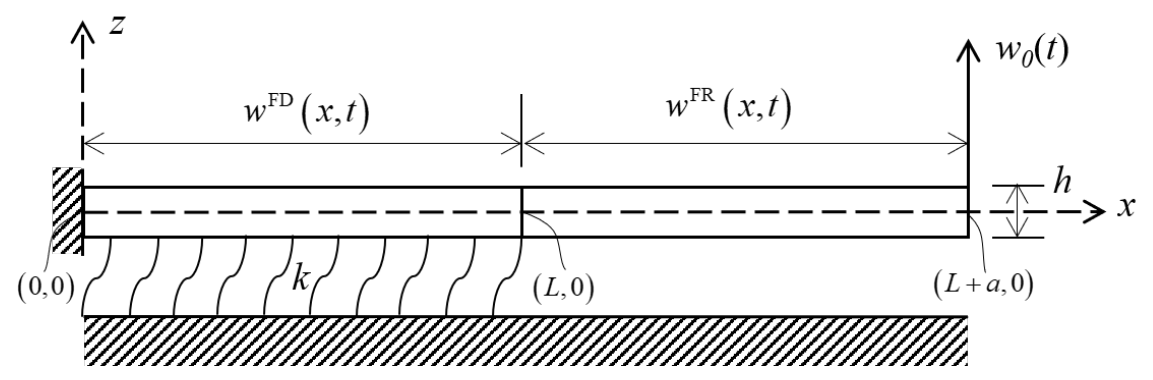

Fig. 1. Schematic of beam partially supported on elastic foundation

Note that the structure shown in Fig. 1 also represents a symmetric DCB, as well as a thin layer on a thick substrate (shown in Fig. 2). The theory to be developed therefore applies to these structures without modification (except for a factor of two in the DCB case). Furthermore, with appropriate modifications to the derivation that maintain the underlying principles and theory, the structure shown in Fig. 1 can also be used to represent embedded cracks in straight beam structures, axisymmetric cracks in circular plates and annuli, etc.
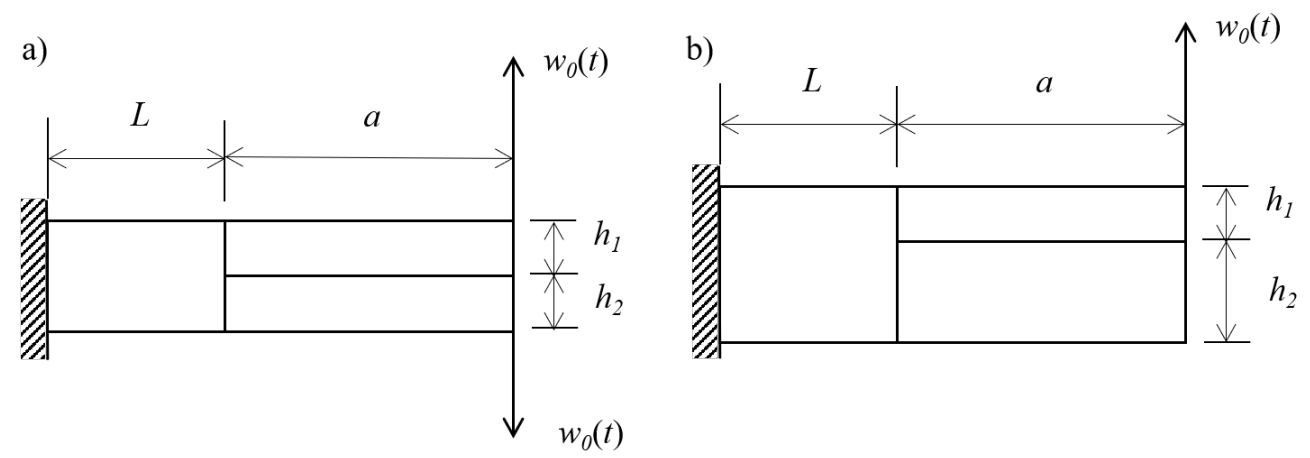

Fig. 2. (a) Symmetric double cantilever beam with $h_{1}=h_{2}=h$; (b) thin layer on thick substrate with $h_{1}=h$ and $h_{1} \square h_{2}$.

The conservation of energy for an elastic structure with a crack of area $A_{0}$ is

$$
W_{\mathrm{ext}}=U+K+S+\Gamma,
$$

where $W_{\text {ext }}$ is the time-accumulated work done by the external forces; $U$ and $K$ are the instantaneous strain energy and the kinetic energy of the beam, respectively; $S$ is the instantaneous strain energy stored in the elastic foundation; and $\Gamma$ is the time-accumulated energy, dissipated from the whole system in opening the crack. At time $t=0$, the system is in its initial condition and all the energy terms are zero. Under displacement control, the applied displacement is held during crack opening and so $d W_{\text {ext }} / d A_{0}=0$. The energy dissipated from the system in incrementing the crack area by $d A_{0}$ is therefore the reduction in total mechanical 
energy of the system, which is $-d(U+K+S)$. A new quantity $\Pi$ is therefore defined here as the total mechanical energy at a given time $t$ that could potentially be dissipated from the system during crack growth, that is,

$$
\Pi=U+K+S
$$

\subsection{Dynamic transverse response of beam partially supported on elastic foundation}

\subsubsection{Deflection assumptions}

By introducing shifting functions [13], the dynamic transverse deflection of the beam shown in Fig. 1 with applied constant-rate displacement $w_{0}(t)=v t$ takes the form of

$$
w^{\mathrm{FD}}(x, t)=w_{\mathrm{fv}}^{\mathrm{FD}}(x, t)+F^{\mathrm{FD}}(x) v t
$$

and

$$
w^{\mathrm{FR}}(x, t)=w_{\mathrm{fv}}^{\mathrm{FR}}(x, t)+F^{\mathrm{FR}}(x) v t,
$$

where $w_{\mathrm{fv}}^{\mathrm{FD}}(x, t)$ and $w_{\mathrm{fv}}^{\mathrm{FR}}(x, t)$ are the free-vibration components of the foundation-supported beam section and the free beam section respectively; and $F^{\mathrm{FD}}(x)$ and $F^{\mathrm{FR}}(x)$ are the corresponding shifting functions. The shifting functions distribute the externally applied displacement along the beam.

The equations of motion [14] for the free vibration of an Euler-Bernoulli beam on an elastic foundation with constant foundation stiffness $k$, and for the free vibration of an EulerBernoulli beam are, respectively,

$$
\begin{gathered}
E I w^{(4)}(x, t)+\rho A \ddot{w}(x, t)+k w(x, t)=0, \\
E I w^{(4)}(x, t)+\rho A \ddot{w}(x, t)=0 .
\end{gathered}
$$

where Lagrange's notation used to represent differentiation with respect to the $x$ coordinate. Other notation has its conventional meaning and is also defined in the table of nomenclature.

For the foundation-supported beam section, the following differential equations for the free vibration component $w_{\mathrm{fv}}^{\mathrm{FD}}(x, t)$ and the corresponding shifting function $F^{\mathrm{FD}}(x)$ are obtained by combining Eqs. (3) and (5) and forcing homogeneous conditions:

$$
\operatorname{EI}_{\mathrm{fv}}^{\mathrm{FD}(4)}(x, t)+\rho A \ddot{w}_{\mathrm{fv}}^{\mathrm{FD}}(x, t)+k w_{\mathrm{fv}}^{\mathrm{FD}}(x, t)=0,
$$




$$
\operatorname{EIF}^{\mathrm{FD}(4)}(x)+k F^{\mathrm{FD}}(x)=0 .
$$

Likewise, using Eqs. (4) and (6) for the free beam section, the differential equations for the free vibration component $w_{\mathrm{fv}}^{\mathrm{FR}}(x, t)$ and the shifting function $F^{\mathrm{FR}}(x)$ are

$$
\begin{gathered}
E I w_{\mathrm{fv}}^{\mathrm{FR}(4)}(x, t)+\rho A \ddot{w}_{\mathrm{fv}}^{\mathrm{FR}}(x, t)=0, \\
F^{\mathrm{FR}(4)}(x)=0 .
\end{gathered}
$$

The corresponding boundary conditions and continuity conditions are given in Appendix A.

\subsubsection{Solution for free vibration component}

By the method of separation of variables, the free-vibration solutions for $w_{\mathrm{fv}}^{\mathrm{FD}}(x, t)$ and $w_{\mathrm{fv}}^{\mathrm{FR}}(x, t)$ from the partial differential equation in Eqs. (7) and (9) are

$$
\begin{aligned}
& w_{\mathrm{fv}}^{\mathrm{FD}}(x, t)=\sum_{i=1}^{\infty} W_{i}^{\mathrm{FD}}(x) T_{i}(t), \\
& w_{\mathrm{fv}}^{\mathrm{FR}}(x, t)=\sum_{i=1}^{\infty} W_{i}^{\mathrm{FR}}(x) T_{i}(t),
\end{aligned}
$$

where $W_{i}(x)$ represents the $i$ th normal mode for the respective beam section, and $T_{i}(t)$ is the time-dependent modal displacement of the $i$ th normal mode. The normal modes, $W_{i}^{\mathrm{FD}}(x)$ and $W_{i}^{\mathrm{FR}}(x)$, can be solved with their respective boundary conditions to give

$$
\begin{gathered}
W_{i}^{\mathrm{FD}}(x)=C_{i 1} \cosh \left(\alpha_{i} x\right) \sin \left(\alpha_{i} x\right)-C_{i 1} \sinh \left(\alpha_{i} x\right) \cos \left(\alpha_{i} x\right)+C_{i 2} \sinh \left(\alpha_{i} x\right) \sin \left(\alpha_{i} x\right), \\
W_{i}^{\mathrm{FR}}(x)=C_{i 3} \sinh \left[\beta_{i}(x-L-a)\right]+C_{i 4} \sin \left[\beta_{i}(x-L-a)\right]
\end{gathered}
$$

in which $4 \alpha_{i}^{4}=k / E I-\omega_{i}^{2} \rho A /(E I)$ and $\beta_{i}^{4}=\omega_{i}^{2} \rho A /(E I)$. Note $C_{i 1}, C_{i 2} C_{i 3}$ and $C_{i 4}$ are coefficients to be determined, and their solution will be described shortly.

The frequency equation is derived by applying the four boundary conditions for the continuity condition at the crack tip, $x=L$, described in Appendix A, to these general solutions for $W_{i}^{\mathrm{FD}}(x)$ and $W_{i}^{\mathrm{FR}}(x)$, which gives 


$$
\left[\begin{array}{cccc}
\left(\begin{array}{c}
\cosh \left(\alpha_{i} L\right) \sin \left(\alpha_{i} L\right) \\
-\sinh \left(\alpha_{i} L\right) \cos \left(\alpha_{i} L\right)
\end{array}\right) & \sinh \left(\alpha_{i} L\right) \sin \left(\alpha_{i} L\right) & \sinh \left(\beta_{i} a\right) & \sin \left(\beta_{i} a\right) \\
2 \alpha_{i} \sinh \left(\alpha_{i} L\right) \sin \left(\alpha_{i} L\right) & \alpha_{i}\left(\begin{array}{l}
\sinh \left(\alpha_{i} L\right) \cos \left(\alpha_{i} L\right) \\
+\cosh \left(\alpha_{i} L\right) \sin \left(\alpha_{i} L\right)
\end{array}\right) & -\beta_{i} \cosh \left(\beta_{i} a\right) & -\beta_{i} \cos \left(\beta_{i} a\right) \\
2 \alpha_{i}^{2}\left(\begin{array}{l}
\sinh \left(\alpha_{i} L\right) \cos \left(\alpha_{i} L\right) \\
+\cosh \left(\alpha_{i} L\right) \sin \left(\alpha_{i} L\right)
\end{array}\right) & 2 \alpha_{i}^{2} \cosh \left(\alpha_{i} L\right) \cos \left(\alpha_{i} L\right) & \beta_{i}^{2} \sinh \left(\beta_{i} a\right) & -\beta_{i}^{2} \sin \left(\beta_{i} a\right) \\
4 \alpha_{i}^{3} \cosh \left(\alpha_{i} L\right) \cos \left(\alpha_{i} L\right) & 2 \alpha_{i}^{3}\left(\begin{array}{l}
\sinh \left(\alpha_{i} L\right) \cos \left(\alpha_{i} L\right) \\
-\cosh \left(\alpha_{i} L\right) \sin \left(\alpha_{i} L\right)
\end{array}\right) & -\beta_{i}^{3} \cosh \left(\beta_{i} a\right) & \beta_{i}^{3} \cos \left(\beta_{i} a\right)
\end{array}\right]=\left(\begin{array}{l}
0 \\
C_{i 2} \\
C_{i 3} \\
C_{i 4}
\end{array}\right) .
$$

For this homogeneous system of linear equations to have non-zero solutions, the determinant of the coefficient matrix must be zero, and this gives the frequency equation. Let $D_{i}$ be the determinant of coefficient matrix of Eq. (15). From $D_{i}=0$, the wavenumber $\beta_{i}$ can be determined and thus angular frequency of each mode is $\omega_{i}=\beta_{i}^{2} \sqrt{E I /(\rho A)}$.

The orthogonality condition is derived in Appendix B as

$$
\int_{0}^{L} \rho A W_{i}^{\mathrm{FD}}(x) W_{j}^{\mathrm{FD}}(x) d x+\int_{L}^{L+a} \rho A W_{i}^{\mathrm{FR}}(x) W_{j}^{\mathrm{FR}}(x) d x=\delta_{i j} .
$$

By applying Gaussian elimination to Eq. (15), the coefficients $C_{i 1}, C_{i 2}$ and $C_{i 3}$ can be expressed linearly in terms of $C_{i 4}$. Then, by substituting the general solutions in Eqs. (13) and (14) into the orthogonality condition in Eq. (16), $C_{i 4}$ can be determined, and therefore $C_{i 1}, C_{i 2}$ and $C_{i 3}$ also.

Returning to the time-dependent modal displacement of the $i$ th normal mode $T_{i}(t)$ in Eqs. (11) and (12), the general solution for $T_{i}(t)$ is

$$
T_{i}(t)=\frac{\dot{T}_{i}(0)}{\omega_{i}} \sin \left(\omega_{i} t\right)+T_{i}(0) \cos \left(\omega_{i} t\right),
$$

where $T_{i}(0)$ and $\dot{T}_{i}(0)$ are the initial values of the modal displacement and modal velocity of the $i$ th normal mode. They are derived in Appendix $\mathrm{C}$, giving $T_{i}(0)=0$ and $\dot{T}_{i}(0)$ as

$$
\begin{aligned}
\dot{T}_{i}(0) & =-v\left[\int_{0}^{L} \rho A W_{i}^{\mathrm{FD}}(x) F^{\mathrm{FD}}(x) d x+\int_{L}^{L+a} \rho A W_{i}^{\mathrm{FR}}(x) F^{\mathrm{FR}}(x) d x\right] \\
& =-v H_{i}
\end{aligned}
$$

where

$$
H_{i}=\int_{0}^{L} \rho A W_{i}^{\mathrm{FD}}(x) F^{\mathrm{FD}}(x) d x+\int_{L}^{L+a} \rho A W_{i}^{\mathrm{FR}}(x) F^{\mathrm{FR}}(x) d x .
$$


The quantity $H_{i}$ represents the coupling of free vibration and the applied constant opening rate, that is, the response of the free vibration of the beam to the applied excitation. It is shown in Section 2.1.3 that the shifting functions, $F^{\mathrm{FD}}(x)$ and $F^{\mathrm{FR}}(x)$, are independent of the applied velocity $v$; therefore, $H_{i}$ is also independent of the applied velocity and is instead an inherent property of the beam configuration.

\subsubsection{Shifting functions}

The shifting functions are obtained by solving the ordinary differential equations in Eqs. (8) and (10), together with the available boundary conditions, including the continuity conditions at the crack tip. The general solution for $F^{\mathrm{FD}}(x)$ and $F^{\mathrm{FR}}(x)$ are, respectively,

$$
\begin{gathered}
F^{\mathrm{FD}}(x)=P_{1} \cosh (\gamma x) \sin (\gamma x)-P_{1} \sinh (\gamma x) \cos (\gamma x)+P_{2} \sinh (\gamma x) \sin (\gamma x), \\
F^{\mathrm{FR}}(x)=P_{3}(x-L-a)^{3}+P_{4}(x-L-a)+1,
\end{gathered}
$$

where $4 \gamma^{4}=k / E I$, and where the solutions for the coefficients, $P_{1}, P_{2}, P_{3}$ and $P_{4}$, are given in Appendix D.

The shifting function solutions, given in Eqs. (20) and (21) together with Eqs. (D2) to (D5), show that they are independent of the applied velocity $v$. This indicates that the shifting functions, which represent the distribution of the externally applied displacement along the beam, are inherent properties of the beam configuration.

The combined results from Sections 2.1.1 to 2.1.3 give the total deflections for foundationsupported beam and free beam sections in Eqs. (3) and (4) as

$$
\begin{aligned}
w^{\mathrm{FD}}(x, t) & =w_{\mathrm{fv}}^{\mathrm{FD}}(x, t)+F^{\mathrm{FD}}(x) v t \\
& =v\left\{-\sum_{i=1}^{\infty}\left[\frac{H_{i}}{\omega_{i}} W_{i}^{\mathrm{FD}}(x) \sin \left(\omega_{i} t\right)\right]+F^{\mathrm{FD}}(x) t\right\}, \\
w^{\mathrm{FR}}(x, t) & =w_{\mathrm{fv}}^{\mathrm{FR}}(x, t)+F^{\mathrm{FR}}(x) v t \\
& =v\left\{-\sum_{i=1}^{\infty}\left[\frac{H_{i}}{\omega_{i}} W_{i}^{\mathrm{FR}}(x) \sin \left(\omega_{i} t\right)\right]+F^{\mathrm{FR}}(x) t\right\} .
\end{aligned}
$$

It is seen that the beam system's response is proportional to the externally applied constant opening velocity $v$. The terms contained in the braces are determined by the beam configuration alone. 


\subsection{Energy balance}

\subsubsection{Strain energy of beam}

The strain energy of a thin beam section is calculated using $U=\int M^{2}(x, t) d x /(2 E I)$, where $M(x, t)=E I w^{(2)}(x, t)$, which is the internal bending moment. The strain energy stored in the foundation-supported beam section is therefore

$$
U^{\mathrm{FD}}=\frac{1}{2} E I\left\{\int_{0}^{L}\left[w_{\mathrm{fv}}^{\mathrm{FD}(2)}(x, t)\right]^{2} d x+2 \int_{0}^{L}\left[w_{\mathrm{fv}}^{\mathrm{FD}(2)}(x, t) F^{\mathrm{FD}(2)}(x) v t\right]^{2} d x+\int_{0}^{L}\left[F^{\mathrm{FD}(2)}(x) v t\right]^{2} d x\right\},
$$

and the strain energy stored in the free beam section is

$$
U^{\mathrm{FR}}=\frac{1}{2} E I\left\{\int_{L}^{L+a}\left[w_{\mathrm{fv}}^{\mathrm{FR}(2)}(x, t)\right]^{2} d x+2 \int_{L}^{L+a}\left[w_{\mathrm{fv}}^{\mathrm{FR}(2)}(x, t) F^{\mathrm{FR}(2)}(x) v t\right]^{2} d x+\int_{L}^{L+a}\left[F^{\mathrm{FR}(2)}(x) v t\right]^{2} d x\right\} .
$$

Let $U_{\mathrm{loc}}^{\mathrm{FD}}, U_{\mathrm{cp}}^{\mathrm{FD}}$ and $U_{\mathrm{st}}^{\mathrm{FD}}$ correspond in order to each of the three terms in Eq. (24), representing the strain energy due to the localized free vibration, the strain energy due to coupling of the localized vibration and the static motion, and the strain energy due to the static motion, respectively. Similar definitions are applied to Eq. (25), with the corresponding strain energy terms being $U_{\mathrm{loc}}^{\mathrm{FR}}, U_{\mathrm{cp}}^{\mathrm{FR}}$ and $U_{\mathrm{st}}^{\mathrm{FR}}$.

The total strain energy of the whole beam is therefore $U=U^{\mathrm{FD}}+U^{\mathrm{FR}}$, which can then be partitioned into the total strain energy due to the localized vibration, $U_{\mathrm{loc}}=U_{\mathrm{loc}}^{\mathrm{FD}}+U_{\mathrm{loc}}^{\mathrm{FR}}$; the total strain energy due to coupling between the localized vibration and the static motion, $U_{\mathrm{cp}}=U_{\mathrm{cp}}^{\mathrm{FD}}+U_{\mathrm{cp}}^{\mathrm{FR}}$; and the total strain energy due to the static motion, $U_{\mathrm{st}}=U_{\mathrm{st}}^{\mathrm{FD}}+U_{\mathrm{st}}^{\mathrm{FR}}$.

$$
\begin{aligned}
U & =U^{\mathrm{FD}}+U^{\mathrm{FR}} \\
& =\left(U_{\mathrm{loc}}^{\mathrm{FD}}+U_{\mathrm{cp}}^{\mathrm{FD}}+U_{\mathrm{st}}^{\mathrm{FD}}\right)+\left(U_{\mathrm{loc}}^{\mathrm{FR}}+U_{\mathrm{cp}}^{\mathrm{FR}}+U_{\mathrm{st}}^{\mathrm{FR}}\right) \\
& =\left(U_{\mathrm{loc}}^{\mathrm{FD}}+U_{\mathrm{loc}}^{\mathrm{FR}}\right)+\left(U_{\mathrm{cp}}^{\mathrm{FD}}+U_{\mathrm{cp}}^{\mathrm{FR}}\right)+\left(U_{\mathrm{st}}^{\mathrm{FD}}+U_{\mathrm{st}}^{\mathrm{FR}}\right) \\
& =U_{\mathrm{loc}}+U_{\mathrm{cp}}+U_{\mathrm{st}} .
\end{aligned}
$$

The total strain energy due to the localized vibration is

$$
U_{\mathrm{loc}}=\frac{1}{2} E I \int_{0}^{L}\left[w_{\mathrm{fv}}^{\mathrm{FD}(2)}(x, t)\right]^{2} d x+\frac{1}{2} E I \int_{L}^{L+a}\left[w_{\mathrm{fv}}^{\mathrm{FR}(2)}(x, t)\right]^{2} d x .
$$

By expanding and rearranging Eq. (27) (see Appendix E), the total strain energy due to the localized vibration $U_{\text {loc }}$ can be expressed as the sum of strain energy contributions from 
individual vibration modes $U_{\text {loc/ }}$ and strain energy contributions from coupling between vibration modes $U_{\text {loc/C }}$. This is expressed as

$$
U_{\mathrm{loc}}=U_{\mathrm{loc} / \mathrm{S}}+U_{\mathrm{loc} / \mathrm{C}},
$$

where

$$
\begin{gathered}
U_{\mathrm{loc} / \mathrm{S}}=\frac{1}{2} E I v^{2} \sum_{i=1}^{\infty} \frac{H_{i}^{2}}{\omega_{i}^{2}} \sin ^{2}\left(\omega_{i} t\right)\left\{\int_{0}^{L}\left[W_{i}^{\mathrm{FD}(2)}(x)\right]^{2} d x+\int_{L}^{L+a}\left[W_{i}^{\mathrm{FR}(2)}(x)\right]^{2} d x\right\}, \\
U_{\mathrm{loc} / \mathrm{C}}=E I v^{2} \lim _{n \rightarrow \infty} \sum_{i=1}^{n-1}\left\{\begin{array}{l}
\int_{0}^{L}\left[W_{i}^{\mathrm{FD}(2)}(x) \frac{H_{i}}{\omega_{i}} \sin \left(\omega_{i} t\right) \sum_{j=i+1}^{n} W_{j}^{\mathrm{FD}(2)}(x) \frac{H_{j}}{\omega_{j}} \sin \left(\omega_{j} t\right)\right] d x \\
+\int_{L}^{L+a}\left[W_{i}^{\mathrm{FR}(2)}(x) \frac{H_{i}}{\omega_{i}} \sin \left(\omega_{i} t\right) \sum_{j=i+1}^{n} W_{j}^{\mathrm{FR}(2)}(x) \frac{H_{j}}{\omega_{j}} \sin \left(\omega_{j} t\right)\right] d x
\end{array}\right\} .
\end{gathered}
$$

The total strain energy due to coupling between the localized vibration and the static motion, that is, $U_{\mathrm{cp}}=U_{\mathrm{cp}}^{\mathrm{FD}}+U_{\mathrm{cp}}^{\mathrm{FR}}$, is

$$
U_{\mathrm{cp}}=E I \int_{0}^{L}\left[w_{\mathrm{fv}}^{\mathrm{FD}(2)}(x, t) F^{\mathrm{FD}(2)}(x) v t\right] d x+E I \int_{L}^{L+a}\left[w_{\mathrm{fv}}^{\mathrm{FR}(2)}(x, t) F^{\mathrm{FR}(2)}(x) v t\right] d x .
$$

The total strain energy due to the static motion, that is, $U_{\mathrm{st}}=U_{\mathrm{st}}^{\mathrm{FD}}+U_{\mathrm{st}}^{\mathrm{FR}}$, is

$$
\begin{aligned}
U_{\mathrm{st}} & =U_{\mathrm{st}}^{\mathrm{FD}}+U_{\mathrm{st}}^{\mathrm{FR}} \\
& =\frac{1}{2} E I \int_{0}^{L}\left[F^{\mathrm{FD}(2)}(x) v t\right]^{2} d x+\frac{1}{2} E I \int_{L}^{L+a}\left[F^{\mathrm{FR}(2)}(x) v t\right]^{2} d x .
\end{aligned}
$$

\subsubsection{Kinetic energy of beam}

The transverse velocities of the foundation-supported beam and the free beam sections are $\dot{w}^{\mathrm{FD}}(x, t)=\dot{w}_{\mathrm{fv}}^{\mathrm{FD}}(x, t)+F^{\mathrm{FD}}(x) v$ and $\dot{w}^{\mathrm{FR}}(x, t)=\dot{w}_{\mathrm{fv}}^{\mathrm{FR}}(x, t)+F^{\mathrm{FR}}(x) v$. The kinetic energy of a thin beam section is calculated using $K=\rho A / 2 \int_{0}^{L}[\dot{w}(x, t)]^{2} d x$, and, therefore, the corresponding kinetic energies are

$$
\begin{gathered}
K^{\mathrm{FD}}=\frac{1}{2} \rho A\left\{\int_{0}^{L}\left[\dot{w}_{\mathrm{fv}}^{\mathrm{FD}}(x, t)\right]^{2} d x+2 \int_{0}^{L}\left[\dot{w}_{\mathrm{fv}}^{\mathrm{FD}}(x, t) F^{\mathrm{FD}}(x) v\right] d x+\int_{0}^{L}\left[F^{\mathrm{FD}}(x) v\right]^{2} d x\right\}, \\
K^{\mathrm{FR}}=\frac{1}{2} \rho A\left\{\int_{L}^{L+a}\left[\dot{w}_{\mathrm{fv}}^{\mathrm{FR}}(x, t)\right]^{2} d x+2 \int_{L}^{L+a}\left[\dot{w}_{\mathrm{fv}}^{\mathrm{FR}}(x, t) F^{\mathrm{FR}}(x) v\right] d x+\int_{L}^{L+a}\left[F^{\mathrm{FR}}(x) v\right]^{2} d x\right\} .
\end{gathered}
$$

Let $K_{\mathrm{loc}}^{\mathrm{FD}}, K_{\mathrm{cp}}^{\mathrm{FD}}$ and $K_{\mathrm{st}}^{\mathrm{FD}}$ correspond in order to each of the three terms in Eq. (33), representing the kinetic energy due to the localized free vibration, the kinetic energy due to the 
coupling of the localized vibration and the static motion, and the kinetic energy due to the static motion, respectively. Similar definitions are applied to Eq. (34) with the corresponding kinetic energy terms being $K_{\mathrm{loc}}^{\mathrm{FR}}, K_{\mathrm{cp}}^{\mathrm{FR}}$ and $K_{\mathrm{st}}^{\mathrm{FR}}$.

The total kinetic energy of the whole beam is therefore $K=K^{\mathrm{FD}}+K^{\mathrm{FR}}$, which can be partitioned into the total kinetic energy due to the localized vibration, $K_{\mathrm{loc}}=K_{\mathrm{loc}}^{\mathrm{FD}}+K_{\mathrm{loc}}^{\mathrm{FR}}$; the total kinetic energy due to coupling between the localized vibration and the static motion, $K_{\mathrm{cp}}=K_{\mathrm{cp}}^{\mathrm{FD}}+K_{\mathrm{cp}}^{\mathrm{FR}}$; and the total kinetic energy due to static motion, $K_{\mathrm{st}}=K_{\mathrm{st}}^{\mathrm{FD}}+K_{\mathrm{st}}^{\mathrm{FR}}$.

$$
K=K_{\mathrm{loc}}+K_{\mathrm{cp}}+K_{\mathrm{st}} .
$$

The total kinetic energy due to the localized vibration is

$$
K_{\mathrm{loc}}=\frac{1}{2} \rho A \int_{0}^{L}\left[\dot{w}_{\mathrm{fv}}^{\mathrm{FD}}(x, t)\right]^{2} d x+\frac{1}{2} \rho A \int_{L}^{L+a}\left[\dot{w}_{\mathrm{fv}}^{\mathrm{FR}}(x, t)\right]^{2} d x .
$$

Like the case for the strain energy due to localized vibration $U_{\text {loc }}$ in Eq. (27), and by the equivalent derivation for Eq. (28) (see Appendix E), the total kinetic energy due to localized vibration can be expressed as the sum of kinetic energy contributions from each individual vibration mode $K_{\text {loc/s }}$ and kinetic energy contributions from coupling between vibration modes $K_{\text {loc/C }}$. In this case, however, the orthogonality of free vibration condition in Eq. (16) can also be applied, allowing $K_{\mathrm{loc} / \mathrm{S}}$ and $K_{\mathrm{loc} / \mathrm{C}}$ to simplify further to $K_{\mathrm{loc} / \mathrm{S}}=v^{2} / 2 \sum_{i=1}^{\infty} H_{i}^{2} \cos ^{2}\left(\omega_{i} t\right)$ and $K_{\mathrm{loc} / \mathrm{C}}=0$, giving

$$
K_{\mathrm{loc}}=\frac{1}{2} v^{2} \sum_{i=1}^{\infty} H_{i}^{2} \cos ^{2}\left(\omega_{i} t\right) .
$$

The total kinetic energy due to coupling between the localized vibration and the static motion, that is, $K_{\mathrm{cp}}=K_{\mathrm{cp}}^{\mathrm{FD}}+K_{\mathrm{cp}}^{\mathrm{FR}}$, with the orthogonality of free vibration condition also applied, is

$$
\begin{aligned}
K_{\mathrm{cp}} & =\rho A \int_{0}^{L}\left[\dot{w}_{\mathrm{fv}}^{\mathrm{FD}}(x, t) F^{\mathrm{FD}}(x) v\right] d x+\rho A \int_{L}^{L+a}\left[\dot{w}_{\mathrm{fv}}^{\mathrm{FR}}(x, t) F^{\mathrm{FR}}(x) v\right] d x \\
& =-v^{2} \sum_{i=1}^{\infty} H_{i}^{2} \cos \left(\omega_{i} t\right) .
\end{aligned}
$$

The total kinetic energy due to the static motion, that is, $K_{\mathrm{st}}=K_{\mathrm{st}}^{\mathrm{FD}}+K_{\mathrm{st}}^{\mathrm{FR}}$, is

$$
K_{\mathrm{st}}=\frac{1}{2} \rho A \int_{0}^{L}\left[F^{\mathrm{FD}}(x) v\right]^{2} d x+\frac{1}{2} \rho A \int_{L}^{L+a}\left[F^{\mathrm{FR}}(x) v\right]^{2} d x .
$$




\subsubsection{Strain energy of elastic foundation}

The strain energy of the elastic foundation is

$$
S=\frac{1}{2} k \int_{0}^{L}\left[w^{\mathrm{FD}}(x, t)\right]^{2} d x
$$

which expands to

$$
S=\frac{1}{2} k\left\{\int_{0}^{L}\left[w_{\mathrm{fv}}^{\mathrm{FD}}(x, t)\right]^{2} d x+2 \int_{0}^{L}\left[w_{\mathrm{fv}}^{\mathrm{FD}}(x, t) F^{\mathrm{FD}}(x) v t\right] d x+\int_{0}^{L}\left[F^{\mathrm{FD}}(x) v t\right]^{2} d x\right\} .
$$

Let $S_{\mathrm{loc}}, S_{\mathrm{cp}}$ and $S_{\mathrm{st}}$ correspond in order to each of the three terms in Eq. (41), representing the strain energy of the foundation due to the localized free vibration, the strain energy of the foundation due to the coupling of the localized vibration and the static motion, and the strain energy of the foundation due to the static motion, respectively.

The strain energy stored in the foundation due to the localized vibration is

$$
S_{\mathrm{loc}}=\frac{1}{2} k \int_{0}^{L}\left\{-v \sum_{i=1}^{\infty}\left[\frac{H_{i}}{\omega_{i}} W_{i}^{\mathrm{FD}}(x) \sin \left(\omega_{i} t\right)\right]\right\}^{2} d x .
$$

Like the case for the strain energy due to localized vibration $U_{\text {loc }}$ in Eq. (27), and by the equivalent derivation for Eq. (28) (see Appendix E), the total strain energy of the foundation due to localized vibration can be expressed as the sum of strain energy contributions from each individual vibration mode $S_{\text {loc/S }}$ and strain energy contributions from coupling between vibration modes $S_{\mathrm{loc} / \mathrm{C}}$. This is expressed as

$$
S_{\mathrm{loc}}=S_{\mathrm{loc} / \mathrm{S}}+S_{\mathrm{loc} / \mathrm{C}},
$$

where

$$
\begin{gathered}
S_{\mathrm{loc} / \mathrm{S}}=\frac{1}{2} k v^{2}\left\{\sum_{i=1}^{\infty} \frac{H_{i}^{2}}{\omega_{i}^{2}} \sin ^{2}\left(\omega_{i} t\right) \int_{0}^{L}\left[W_{i}^{\mathrm{FD}}(x)\right]^{2} d x\right\}, \\
S_{\mathrm{loc} / \mathrm{C}}=k v^{2} \lim _{n \rightarrow \infty} \sum_{i=1}^{n-1}\left\{\int_{0}^{L} \frac{H_{i}}{\omega_{i}} W_{i}^{\mathrm{FD}}(x) \sin \left(\omega_{i} t\right) \sum_{j=i+1}^{n}\left[\frac{H_{j}}{\omega_{j}} W_{j}^{\mathrm{FD}}(x) \sin \left(\omega_{j} t\right)\right] d x\right\} .
\end{gathered}
$$

The total strain energy of the foundation due to coupling between the localized vibration and the static motion is

$$
S_{\mathrm{cp}}=-k v^{2} t \sum_{i=1}^{\infty}\left\{\frac{H_{i}}{\omega_{i}} \sin \left(\omega_{i} t\right) \int_{0}^{L}\left[W_{i}^{\mathrm{FD}}(x) F^{\mathrm{FD}}(x)\right] d x\right\} .
$$

The total strain energy of the foundation due to the static motion is 


$$
S_{\mathrm{st}}=\frac{1}{2} k v^{2} t^{2} \int_{0}^{L}\left[F^{\mathrm{FD}}(x)\right]^{2} d x
$$

\subsubsection{Total mechanical energy}

The localized vibration strain-energy contributions from individual vibration modes, that is, $U_{\text {loc/S }}$ in Eq. (29), can be simplified as per Appendix F to

$$
\begin{aligned}
U_{\mathrm{loc} / \mathrm{S}} & =-\frac{1}{2} k v^{2} \sum_{i=1}^{\infty}\left\{\frac{H_{i}^{2}}{\omega_{i}^{2}} \sin ^{2}\left(\omega_{i} t\right) \int_{0}^{L}\left[W_{i}^{\mathrm{FD}}(x)\right]^{2} d x\right\}+\frac{1}{2} v^{2} \sum_{i=1}^{\infty} H_{i}^{2} \sin ^{2}\left(\omega_{i} t\right) \\
& =-S_{\mathrm{loc} / \mathrm{S}}+\frac{1}{2} v^{2} \sum_{i=1}^{\infty} H_{i}^{2} \sin ^{2}\left(\omega_{i} t\right) .
\end{aligned}
$$

The localized vibration strain-energy contributions from coupling between vibration modes, that is, $U_{\text {loc/C }}$ in Eq. (30), can be simplified as per Appendix F to

$$
\begin{aligned}
U_{\mathrm{loc} / \mathrm{C}} & =-k v^{2} \lim _{n \rightarrow \infty} \sum_{i=1}^{n-1}\left\{\int_{0}^{L} \frac{H_{i}}{\omega_{i}} \sin \left(\omega_{i} t\right) W_{i}^{\mathrm{FD}}(x) \sum_{j=i+1}^{n}\left[W_{j}^{\mathrm{FD}}(x) \frac{H_{j}}{\omega_{j}} \sin \left(\omega_{j} t\right)\right] d x\right\} \\
& =-S_{\mathrm{loc} / \mathrm{C}} .
\end{aligned}
$$

The strain energy due to coupling between the localized vibration and the static motion, that is, $U_{\text {cp }}$ in Eq. (31), can be simplified as per Appendix G to

$$
\begin{aligned}
U_{\mathrm{cp}} & =k v^{2} t \sum_{i=1}^{\infty}\left\{\frac{H_{i}}{\omega_{i}} \sin \left(\omega_{i} t\right) \int_{0}^{L} W_{i}^{\mathrm{FD}}(x) F^{\mathrm{FD}}(x) d x\right\} \\
& =-S_{\mathrm{cp}} .
\end{aligned}
$$

The strain energy due to the static motion, that is, $U_{\text {st }}$ in Eq. (32), can be simplified as per Appendix $\mathrm{H}$ to

$$
\begin{aligned}
U_{\text {st }} & =-\frac{1}{2} E I v^{2} t^{2} F^{\mathrm{FR}(3)}(L+a)-\frac{1}{2} k v^{2} t^{2} \int_{0}^{L}\left[F^{\mathrm{FD}}(x)\right]^{2} d x \\
& =-\frac{1}{2} E I v^{2} t^{2} F^{\mathrm{FR}(3)}(L+a)-S_{\mathrm{st}} .
\end{aligned}
$$

By substituting Eqs. (37) to (39) and Eqs. (48) to (51) into Eq. (2), the total mechanical energy of the system at a given time $t$ that could potentially be dissipated from the system during crack growth is 


$$
\begin{aligned}
\Pi & =-v^{2} \sum_{i=1}^{\infty} H_{i}^{2} \cos \left(\omega_{i} t\right)+\frac{1}{2} v^{2} \sum_{i=1}^{\infty} H_{i}^{2} \\
& -\frac{1}{2} E J^{2} t^{2} F^{\mathrm{FR}(3)}(L+a) \\
& +\frac{1}{2} \rho A \int_{0}^{L}\left[F^{\mathrm{FD}}(x) v\right]^{2} d x+\frac{1}{2} \rho A \int_{L}^{L+a}\left[F^{\mathrm{FR}}(x) v\right]^{2} d x
\end{aligned}
$$

\subsection{Energy release rate}

Considering Eq. (2), the energy that can potentially be dissipated from the system during crack growth comes from the mechanical energy of system. The total mechanical energy is given by $\Pi$ in Eq. (52). Let $\Pi=\Pi_{\text {vib }}+\Pi_{\mathrm{st}}^{\mathrm{U}}+\Pi_{\mathrm{st}}^{\mathrm{K}}$, where

$$
\begin{gathered}
\Pi_{\mathrm{vib}}=-v^{2} \sum_{i=1}^{\infty} H_{i}^{2} \cos \left(\omega_{i} t\right)+\frac{1}{2} v^{2} \sum_{i=1}^{\infty} H_{i}^{2}, \\
\Pi_{\mathrm{st}}^{\mathrm{U}}=-\frac{1}{2} \operatorname{EIv}^{2} t^{2} F^{\mathrm{FR}(3)}(L+a), \\
\Pi_{\mathrm{st}}^{\mathrm{K}}=\frac{1}{2} \rho A \int_{0}^{L}\left[F^{\mathrm{FD}}(x) v\right]^{2} d x+\frac{1}{2} \rho A \int_{L}^{L+a}\left[F^{\mathrm{FR}}(x) v\right]^{2} d x,
\end{gathered}
$$

in which $\Pi_{\text {vib }}$ is the vibrating energy component, $\Pi_{\text {st }}^{\mathrm{U}}$ is the strain energy component due to static motion, and $\Pi_{\mathrm{st}}^{\mathrm{K}}$ is the kinetic energy component due to static motion, which is constant and proportional to $v^{2}$, the square of applied opening velocity.

The dynamic ERR can also be expressed as the sum of respective components, that is, $G=G_{\mathrm{st}}^{\mathrm{U}}+G_{\mathrm{st}}^{\mathrm{K}}+G_{\mathrm{vib}}$, where $G_{\mathrm{st}}^{\mathrm{U}}$ and $G_{\mathrm{st}}^{\mathrm{K}}$ are the ERR components due to the strain energy and the kinetic energy of static motion, respectively, and $G_{\text {vib }}$ is the ERR component due to vibration. Note that $G_{\mathrm{st}}^{\mathrm{U}}$ is the static ERR without any consideration for dynamic effects; and that $G_{\mathrm{st}}^{\mathrm{K}}$ is the kinetic contribution in Refs. [3,4]. These two components can be readily determined as $G_{\mathrm{st}}^{\mathrm{U}}=-d \Pi_{\mathrm{st}}^{\mathrm{U}} / d A_{0}$ and $G_{\mathrm{st}}^{\mathrm{K}}=-d \Pi_{\mathrm{st}}^{\mathrm{K}} / d A_{0}$. This work determines the vibrating dynamic ERR $G_{\text {vib }}$ for the first time, which includes higher-order vibration. In general, $G_{\mathrm{vib}} \neq-d \Pi_{\mathrm{vib}} / d A_{0}$, and instead the dispersive propagation of flexural waves must be considered to derive the energy flux through a contour surrounding the crack tip.

\subsubsection{ERR component due to vibration}

According to Ref. [15], the dynamic ERR can be calculated as 


$$
G=\frac{F(\Omega)}{\dot{A}_{0}},
$$

where $F(\Omega)$ is the instantaneous rate of energy flow through a contour $\Omega$ surrounding the crack tip, also known as the energy flux. The ERR component due to vibration $G_{\text {vib }}$ is therefore

$$
G_{\mathrm{vib}}=\frac{F_{\mathrm{vib}}(\Omega)}{\dot{A}_{0}}=\frac{E_{\text {tot }} C_{1}^{\mathrm{p}}}{\dot{A}_{0}},
$$

where $F_{\text {vib }}(\Omega)$ is the energy flux through the contour $\Omega$ due to vibration. This energy flux can be determined by $F_{\text {vib }}(\Omega)=E_{\text {tot }} C_{1}^{\mathrm{p}}$, where $E_{\text {tot }}$ is the total energy density and $C_{1}^{\mathrm{p}}$ is the phase speed of the first vibration mode's wave. For flexural waves in beams, which are dispersive, the energy of a wave propagates at its group velocity [16,17], but when considering the total energy density, it propagates with the phase speed of the first vibration mode's wave because this wave modulates all higher frequency waves [16].

To determine the total energy density $E_{\text {tot }}$, and hence via Eq. (56), the ERR component due to vibration $G_{\text {vib }}$, consider $\Pi_{\text {vib }}$ in Eq. (53) as having two components, $\Pi_{\text {vib }}=\Pi_{\mathrm{C}}+\Pi_{\mathrm{S}}$, which give rise to $E_{\text {tot }}$ with two corresponding components, $E_{\text {tot }}=E_{\mathrm{C}}+E_{\mathrm{S}}$. The $E_{\mathrm{C}}$ component of $E_{\text {tot }}$ comes from $\Pi_{\mathrm{C}}$, which is the first term in Eq. (53) and is the kinetic energy due to coupling of localized vibration and static motion. For the $i$ th vibration mode's wave, the energy flux from coupling of localized vibration and static motion is $F_{\mathrm{C} i}=E_{\mathrm{C} i} C_{i}^{\mathrm{g}}$, and $F_{\mathrm{C} i}=-d \Pi_{\mathrm{C} i} / d t$. Note that for reasons that will soon become clear, $E_{\mathrm{C}} \neq \sum_{i=1}^{\infty} E_{\mathrm{C} i}$, and so also, $F_{\mathrm{C}} \neq \sum_{i=1}^{\infty} F_{\mathrm{C} i}$ and $F_{\text {vib }} \neq-d \Pi_{\text {vib }} / d t$. The $E_{\mathrm{S}}$ component of $E_{\mathrm{tot}}$ comes from $\Pi_{\mathrm{S}}$, which is the second term in Eq. (53) and is the strain and kinetic energy due to localized vibration. For the $i$ th vibration mode's wave, the energy flux from the strain and kinetic energy due to localized vibration is $F_{\mathrm{S} i}=E_{\mathrm{S} i} C_{i}^{\mathrm{g}}$, and $F_{\mathrm{S} i}=-d \Pi_{\mathrm{S} i} / d t$.

Now consider the net contribution from all the vibration modes' waves to $E_{\mathrm{C}}$. If the foundation stiffness $k$ is relatively large, then the shifting function for the foundationsupported beam section is approximately zero. Therefore, by simplifying Eq. (38) with $F^{\mathrm{FD}}(x) \approx 0$, expanding $\dot{w}_{\mathrm{fv}}^{\mathrm{FR}}(x, t)$ using Eq. (12), and substituting in $\Pi_{\mathrm{C}}$, which is the first term of Eq. (53), then $\Pi_{C i}$ is obtained as 


$$
\Pi_{\mathrm{C} i}=K_{\mathrm{cp} i} \approx-\rho A v^{2} \int_{L}^{L+a}\left\{\sum_{i=1}^{\infty}\left[H_{i} W_{i}^{\mathrm{FR}}(x) F^{\mathrm{FR}}(x)\right]\right\} d x \cos \left(\omega_{i} t\right) .
$$

The integrand of Eq. (58) represents the spatial coupling of the normal modes and the shifting function due to velocity coupling between localized vibration and applied displacement. Figure 3 shows a small contour $\Omega$ around the crack tip with $\varepsilon \square a$.

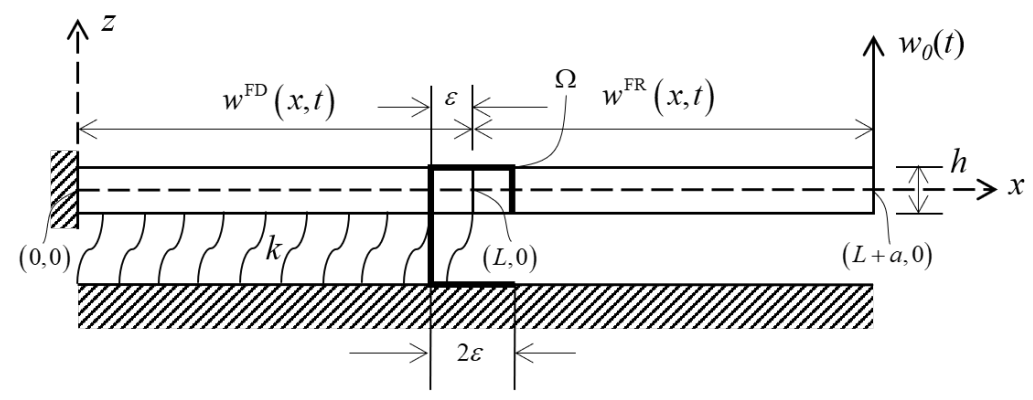

Fig. 3. Crack tip contour $\Omega$

Inside this contour in a region behind the crack tip, that is, $L-\varepsilon \leq x \leq L+\varepsilon$, it can be shown that $F^{\mathrm{FR}}(x)>0$ and $W_{i}^{\mathrm{FR}}(x)>0$, but that the sign of $H_{i}$ alternates with vibration mode numbers with $H_{i}<0$ for odd modes and $H_{i}>0$ for even modes. This can be seen by referring to [12], which has the effective boundary condition of a clamp at the crack tip: $H_{i}$ in this work is in proportion to $\Lambda_{i}$ in Ref. [12], which alternates with vibration mode numbers as described above. The integrand of Eq. (58) is therefore negative for odd modes, which means that the corresponding $\Pi_{\mathrm{C} i}$ reduces the energy density, and is positive for even modes, which means that the corresponding $\Pi_{\mathrm{C} i}$ increases the energy density. Therefore, the total energy density due to $\Pi_{\mathrm{C} i}$ is

$$
E_{\mathrm{C}}=\sum_{i=1}^{\infty}(-1)^{i} E_{\mathrm{C} i}
$$

The physical interpretation of Eq. (59) is that, near to the crack tip, when the transverse velocity of the free vibration is in the same direction as the applied velocity, the energy flux tends to open the crack and increase the total energy density and ERR. When, however, near to the crack tip, the transverse velocity of the free vibration is in the opposite direction to the applied velocity, the energy flux tends to close the crack and decrease the total energy density and ERR.

Likewise, the energy density of $E_{\mathrm{S}}$ due to $\Pi_{\mathrm{S}}$ can be determined by $E_{\mathrm{S}}=\sum_{i=1}^{\infty}(-1)^{i} E_{\mathrm{S} i}$ with an alternating $H_{i}$. The total energy density due to vibration is therefore 


$$
E_{\mathrm{tot}}=\sum_{i=1}^{\infty}(-1)^{i} E_{\mathrm{C} i}+\sum_{i=1}^{\infty}(-1)^{i} E_{\mathrm{S} i} .
$$

By substituting Eq. (60) into Eq. (57), the ERR component due to vibration is

$$
\begin{aligned}
G_{\mathrm{vib}} & =\frac{C_{1}^{\mathrm{p}}}{\dot{A}_{0}}\left[\sum_{i=1}^{\infty}(-1)^{i} \frac{F_{\mathrm{C} i}}{C_{i}^{\mathrm{g}}}+\sum_{i=1}^{\infty}(-1)^{i} \frac{F_{\mathrm{S} i}}{C_{i}^{\mathrm{g}}}\right] \\
& =-\frac{C_{1}^{\mathrm{p}}}{d A_{0} / d t}\left[\sum_{i=1}^{\infty}(-1)^{i} \frac{d \Pi_{\mathrm{C} i} / d t}{C_{i}^{\mathrm{g}}}+\sum_{i=1}^{\infty}(-1)^{i} \frac{d \Pi_{\mathrm{S} i} / d t}{C_{i}^{\mathrm{g}}}\right] \\
& =-\sum_{i=1}^{\infty}(-1)^{i} \frac{d \Pi_{\mathrm{C} i}}{d A_{0}} \frac{C_{1}^{\mathrm{p}}}{C_{i}^{\mathrm{g}}}-\sum_{i=1}^{\infty}(-1)^{i} \frac{d \Pi_{\mathrm{S} i}}{d A_{0}} \frac{C_{1}^{\mathrm{p}}}{C_{i}^{\mathrm{g}}} .
\end{aligned}
$$

The ratio $C_{1}^{\mathrm{p}} / C_{i}^{\mathrm{g}}$ relates the energy flux to the dispersion of flexural waves in beams. Ref. [17] gives $C_{1}^{\mathrm{p}}=\sqrt{\omega_{1}} \sqrt[4]{E I /(\rho A)}$ and $C_{i}^{\mathrm{g}}=2 \sqrt{\omega_{i}} \sqrt[4]{E I /(\rho A)}$, and so $C_{1}^{\mathrm{p}} / C_{i}^{\mathrm{g}}=\sqrt{\omega_{1} /\left(4 \omega_{i}\right)}$.

Eq. (61) can be expanded into the following form:

$$
\begin{aligned}
G_{\mathrm{vib}}= & \frac{v^{2}}{4 b} \sum_{i=1}^{\infty}(-1)^{i} \sqrt{\frac{\omega_{1}}{\omega_{i}}} \frac{d H_{i}}{d a} \cos \left(\omega_{i} t\right)-\frac{v^{2} t}{2 b} \sum_{i=1}^{\infty}(-1)^{i} \sqrt{\frac{\omega_{1}}{\omega_{i}}} H_{i}^{2} \frac{d \omega_{i}}{d a} \sin \left(\omega_{i} t\right) \\
& -\frac{v^{2}}{2 b} \sum_{i=1}^{\infty}(-1)^{i} \sqrt{\frac{\omega_{1}}{\omega_{i}}} \frac{d H_{i}}{d a}
\end{aligned}
$$

The derivation of $d \omega_{i} / d a$ is given in Appendix I. Unless $t$ is small, the first and third terms of Eq. (62) are much smaller than the second term, which increases with time, that is, $G_{\mathrm{vib}} \approx-v^{2} t /(2 b) \sum_{i=1}^{\infty}(-1)^{i} \sqrt{\omega_{1} / \omega_{i}} H_{i}^{2}\left(d \omega_{i} / d a\right) \sin \left(\omega_{i} t\right)$. Moreover Chen et al. [12] showed that the first and third terms are always very small in comparison to the second term for the case of a built-in beam. FEM verification studies in Section 3 also confirm that the approximation is appropriate.

\subsubsection{ERR components due to static motion}

The ERR component due to the strain energy of static motion is

$$
G_{\mathrm{st}}^{\mathrm{U}}=-\frac{d \Pi_{\mathrm{st}}^{\mathrm{U}}}{d A_{0}}=\frac{E I v^{2} t^{2}}{2 b} \frac{d F^{\mathrm{FR}(3)}(L+a)}{d a},
$$

where, from Eq. (21), $F^{\mathrm{FR}(3)}(L+a)=6 P_{3}$. Assuming the product of the foundation stiffness $k$ and the length of the foundation-supported beam section $L$ is large enough to satisfy $\tanh (2 \gamma L) \approx 1$ (that is, $\gamma L \gtrsim 3$ so that $\tanh (6) \approx 0.99999$ ), then $P_{3}$ in Eq. (D4) approximates to 


$$
P_{3}=\frac{-\gamma^{3}}{2 a^{3} \gamma^{3}+6 a^{2} \gamma^{2}+6 a \gamma+3}
$$

and $G_{\text {st }}^{\mathrm{U}}$ simplifies to

$$
G_{\mathrm{st}}^{\mathrm{U}}=\frac{9 E I v^{2} t^{2} f_{\mathrm{st}}^{\mathrm{U}}}{2 b a^{4}}
$$

where

$$
f_{\mathrm{st}}^{\mathrm{U}}=\frac{4 a^{4} \gamma^{4}(a \gamma+1)^{2}}{\left(2 a^{3} \gamma^{3}+6 a^{2} \gamma^{2}+6 a \gamma+3\right)^{2}}
$$

For a rigid interface, the foundation stiffness $k$ and $\gamma$ approach infinity, and so $f_{\text {st }}^{\mathrm{U}}=1$ and $G_{\mathrm{st}}^{\mathrm{U}}=9 E I v^{2} t^{2} /\left(2 b a^{4}\right)$. Therefore, $f_{\mathrm{st}}^{\mathrm{U}}$ can be viewed as a static ERR reduction factor for nonrigid linear elastic interfaces.

Based on Eq. (55), the ERR component due to the kinetic energy of static motion, $G_{\mathrm{st}}^{\mathrm{K}}=-d \Pi_{\mathrm{st}}^{\mathrm{K}} / d A_{0}$, is constant and only gives the total ERR a downwards shift. As above, if $\tanh (2 \gamma L) \approx 1$, then $G_{\text {st }}^{\mathrm{K}}$ is

$$
G_{\mathrm{st}}^{\mathrm{K}}=-\frac{33 \rho A v^{2} f_{\mathrm{st}}^{\mathrm{K}}}{280 b}
$$

where

$$
f_{\mathrm{st}}^{\mathrm{K}}=\frac{\left(\begin{array}{c}
88 a^{9} \gamma^{9}+792 a^{8} \gamma^{8}+3168 a^{7} \gamma^{7}+7420 a^{6} \gamma^{6}+11256 a^{5} \gamma^{5} \\
+12180 a^{4} \gamma^{4}+10920 a^{3} \gamma^{3}+8820 a^{2} \gamma^{2}+5040 a \gamma+1260
\end{array}\right)}{11\left(2 a^{3} \gamma^{3}+6 a^{2} \gamma^{2}+6 a \gamma+3\right)^{3}}
$$

It is worth noting that if $a \gamma \gtrsim 4.7$ then $f_{\text {st }}^{\mathrm{K}}>0.995$. For a rigid interface $f_{\mathrm{st}}^{\mathrm{K}}=1$, and this ERR component becomes the same as that given by Smiley and Pipes [3] and Blackman et al. [4].

\subsubsection{Total dynamic energy release rate and dynamic factor}

By summing Eqs. (62), (65), and (67), the total dynamic ERR under the above assumptions is approximately

$$
G=\frac{9 E I v^{2} t^{2} f_{\mathrm{st}}^{\mathrm{U}}}{2 b a^{4}}-\frac{33 \rho A v^{2} f_{\mathrm{st}}^{\mathrm{K}}}{280 b}-\frac{v^{2} t}{2 b} \sum_{i=1}^{\infty}(-1)^{i} \sqrt{\frac{\omega_{1}}{\omega_{i}}} H_{i}^{2} \frac{d \omega_{i}}{d a} \sin \left(\omega_{i} t\right) .
$$

Note that Eq. (69) is for a crack on the interface between a partially supported beam and an elastic foundation; for a DCB, the ERR is simply twice Eq. (69). 
In Eq. (69), the total dynamic effect of last two terms can be grouped together as $G_{\mathrm{dyn}}=G_{\mathrm{st}}^{\mathrm{K}}+G_{\mathrm{vib}}$. A dynamic factor can therefore be defined as

$$
f_{\mathrm{dyn}}=\frac{G_{\mathrm{dyn}}}{G_{\mathrm{st}}^{\mathrm{U}}}=-\frac{11 f_{\mathrm{st}}^{\mathrm{K}}}{420 f_{\mathrm{st}}^{\mathrm{U}}} \frac{\rho A}{E I} \frac{a^{4}}{t^{2}}-\frac{1}{9 f_{\mathrm{st}}^{\mathrm{U}}} \frac{1}{E I} \frac{a^{4}}{t} \sum_{i=1}^{\infty}(-1)^{i} \sqrt{\frac{\omega_{1}}{\omega_{i}}} H_{i}^{2} \sin \left(\omega_{i} t\right) \frac{d \omega_{i}}{d a} .
$$

Dynamic effects arise due to the kinetic energy of static motion and due to vibration. The former was solved by Smiley and Pipes' [3], in which the dynamic factor follows an inverse square law with respect to time and decays very quickly [12]. The latter dynamic effect, the second term of Eq. (70), is the focus of this work. It is therefore called the dynamic factor due to vibration, denoted by $f_{\text {vib }}$, and is the summation of contributions from all vibration modes.

To show the relative contribution from each vibration mode for a rigid interface, a Fourier analysis was conducted using the Fast Fourier Transform algorithm to transform the dynamic factor due to vibration in Eq. (70) from the time domain $(0 \sim 0.04 \mathrm{~s})$ to the frequency domain. For this the geometry and material properties described in Section 3.1 were used. The first ten vibration modes are shown in Fig. 4, with the dynamic factor due to vibration normalized by the dynamic factor due to vibration of the first vibration mode. With increasing vibration mode frequency, the dynamic factor decreases monotonically. The first vibration mode makes the greatest contribution, which drops dramatically over the first five modes. The first five vibration modes are therefore adequate to capture the major contributions to the dynamic ERR.

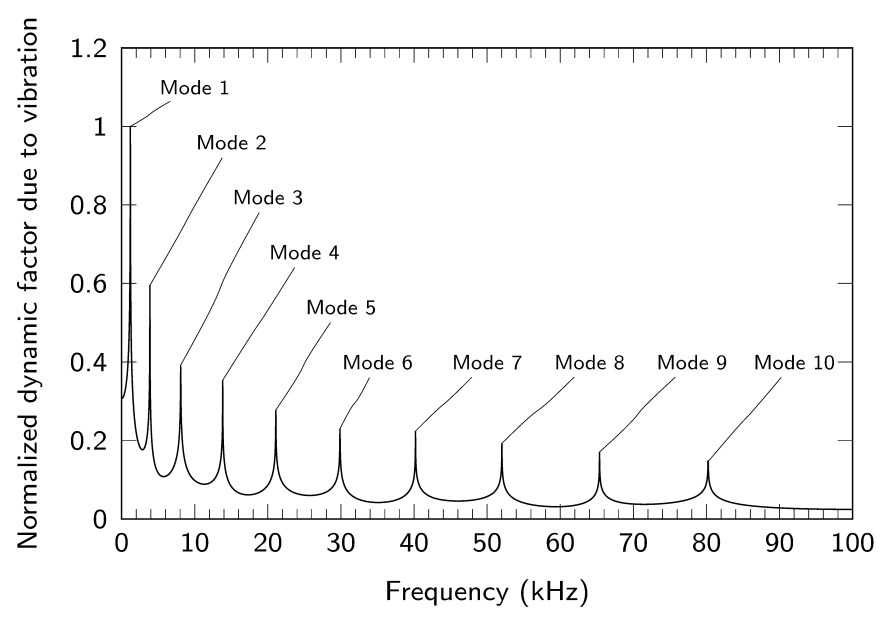

Fig. 4. Normalized dynamic factor due to vibration first ten modes for rigid interface

Now considering the effect of foundation stiffness, Fourier analyses were again conducted on Eq. (70) using four levels of foundation stiffness, namely, $k / E=1,0.1,0.01$ and 0.001 , and with the same geometry and material properties. The results are normalized by the dynamic 
factor due to vibration of the first mode with $k / E=1$ (the numerical values are tabulated in Appendix J). The results are shown in Fig. 5. With decreasing foundation stiffness, the frequency spectra shift to the left, with higher-order vibration modes being more sensitive to the foundation stiffness change. Another characteristic to note is that the contribution from the first vibration mode increases with decreasing foundation stiffness, which indicates that lowerorder vibration modes become even more dominant for less stiff foundations.

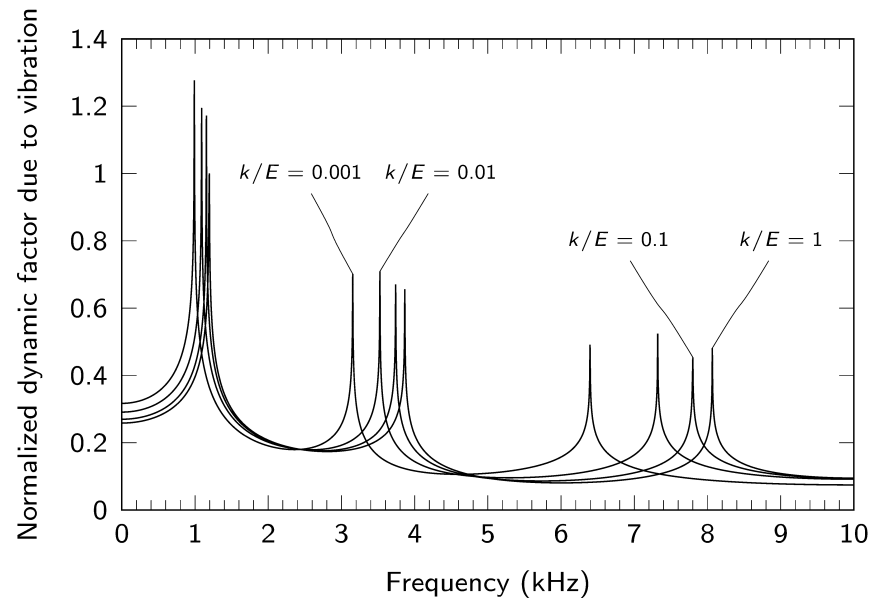

Fig. 5. Normalized dynamic factors due to vibration versus frequency for various foundation stiffnesses

\section{Finite-element-method verification}

The finite-element method (FEM) was used to verify the theory developed in Section 2. Three verification studies were conducted using the geometries shown in Fig. 6:

(1) To verify that the developed theory agrees with FEM simulations for cracks on rigid interfaces, also considering vibration-mode convergence.

(2) To verify that the developed theory agrees with FEM simulations for cracks on non-rigid linear elastic interfaces.

(3) To inspect the fracture mode mixity of a crack between a thin layer and a thick substrate under dynamic loading. 


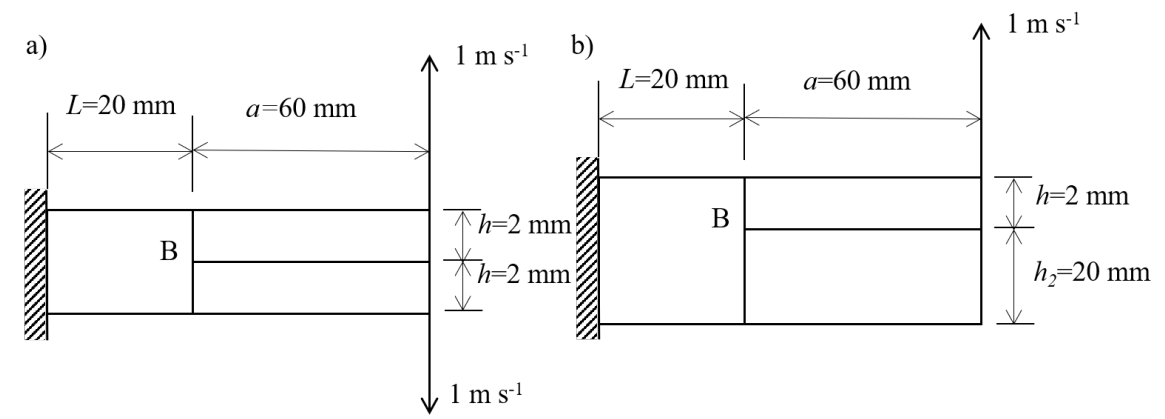

Fig. 6. (a) DCB geometry for FEM verification studies 1 and 2; (b) thin layer on thick substrate geometry for FEM verification study 3 .

\subsection{Rigid interface}

The DCB geometry shown in Fig. 6a with a beam width of $1 \mathrm{~mm}$ was used for the first verification study of a crack on a rigid interface. The Young's modulus was $10 \mathrm{GPa}$, the Poisson's ratio 0.3 , and the density $10^{3} \mathrm{~kg} \mathrm{~m}^{-3}$. A linear 2D FEM model was constructed using four-node plane-stress elements in Abaqus/Explicit (CPS4R) with a global mesh size of $0.2 \mathrm{~mm}$, except for the $1 \times 0.5 \mathrm{~mm}^{2}$ region around the crack tip where the mesh size was refined to $0.05 \mathrm{~mm}$. The total number of elements used was 8616 . The uncracked interface ahead of the crack tip was formed by sharing nodes and no contact was simulated. The virtual crack closure technique (VCCT) was applied to determine the dynamic ERR.

For the developed theory, an appropriate foundation stiffness that represents a rigid interface needs to be used. An interface stiffness of the same order as the beam material's Young's modulus can be regarded as a rigid interface [18], but there is no unanimous agreement on the exact value in the literature (see, for example, Refs. [19,20,21]). For application to composite DCBs, the interfacial stiffness can be determined by $K=\alpha E_{3} / h_{0}$, where $E_{3}$ is the transverse modulus, $h_{0}$ is the thickness of an adjacent sub-laminate, and $\alpha$ is a parameter much large than $1[22]$.

Since the foundation stiffness that represents a rigid interface is not the major focus of this work, a parametric study was conducted using the developed theory to test a range of $k$ values. It was found that, for this verification case, using $k=0.35 E$ provides the best agreement with the FEM results. Note that the rigid interface represents a special case. In general, the relationship between the normal stiffness of the interface $E_{n n}$, which is a measurable material property, and the foundation stiffness used in the theoretical development $k$, is $E_{n n}=0.5 k$. This relationship is a consequence of the symmetric deformation of the DCB and its interface 
under the prescribed loading in comparison to the 'one-sided' deformation of a partially supported beam on an elastic foundation, which the theory is for. Since this relationship is not required for the rigid-interface verification in this section, more details and verification will be given in Section 3.2 for non-rigid interfaces.

The dynamic ERR versus time results from the FEM and the developed theory are compared in Fig. 7, with the black line representing the theory (with $k=0.35 E$ ) and the gray line representing the FEM. The black dashed line represents the ERR from the strain energy of static motion in Eq. (65), that is, the ERR without any dynamic effect. The first one, two, three, four, five, ten, 15 and 20 vibration modes are shown in subfigures a- $\mathrm{h}$ respectively. As more vibration modes are considered in the theory, it becomes in increasingly closer agreement with the FEM in terms of the overall magnitude of ERR, and the frequencies, phases and amplitudes of oscillation. It is seen that the theory with the first five vibration modes (Fig. 7e) is adequate to capture the dynamic ERR very accurately; adding more vibration modes (Fig. 7f-h) provides more detail, but the changes in the amplitude are not significant.

Note that the theory with just the first vibration mode (Fig. 7a) is close to what was reported in Ref. [12] for a fixed support at the crack tip, but with half the ERR amplitude. In that work, however, the developed theory was slightly out-of-phase due to the simplified boundary condition. In this work, this phase difference was substantially corrected by using a partially supported beam and an elastic foundation. 
(a)

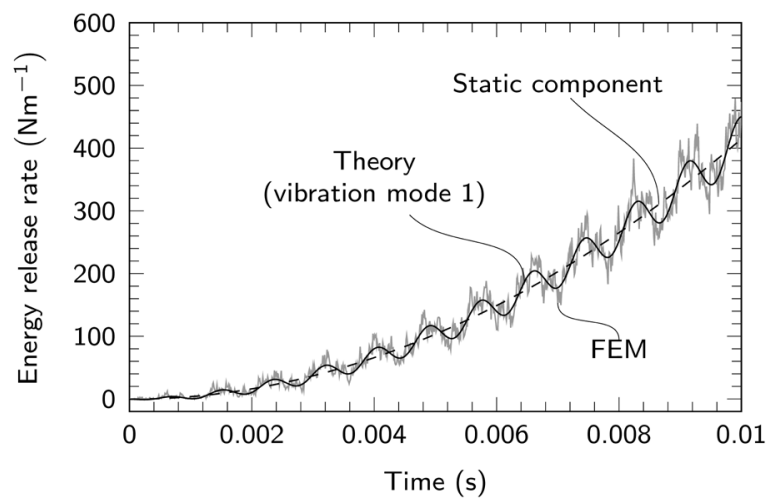

(c)

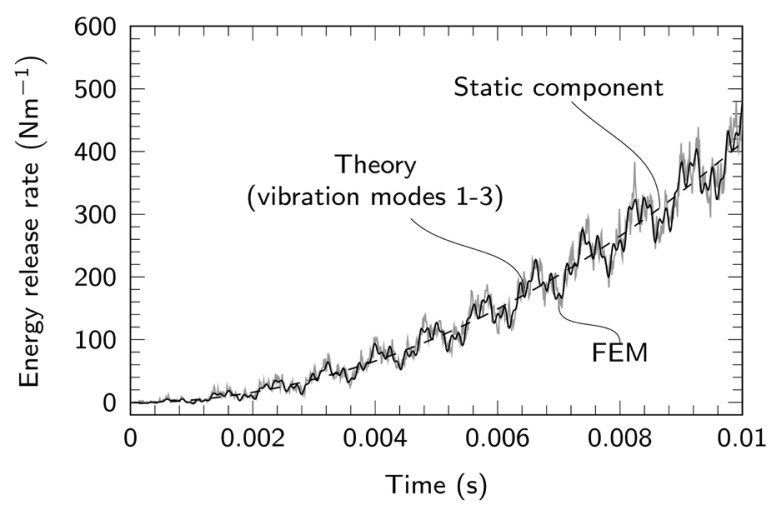

(e)

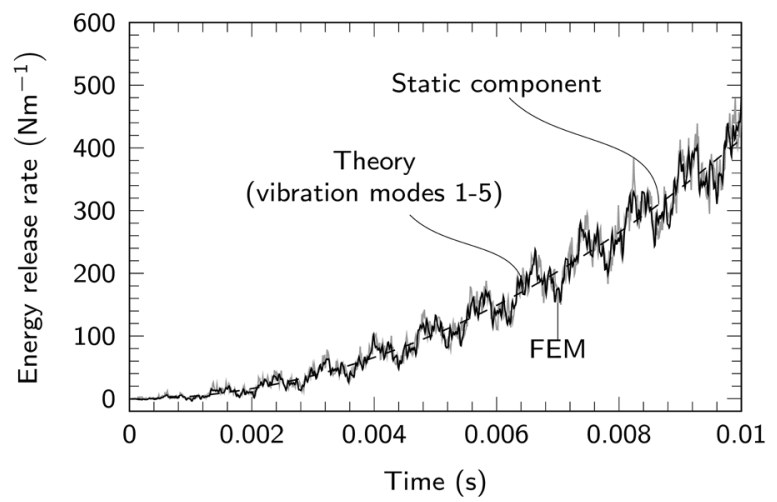

(g)

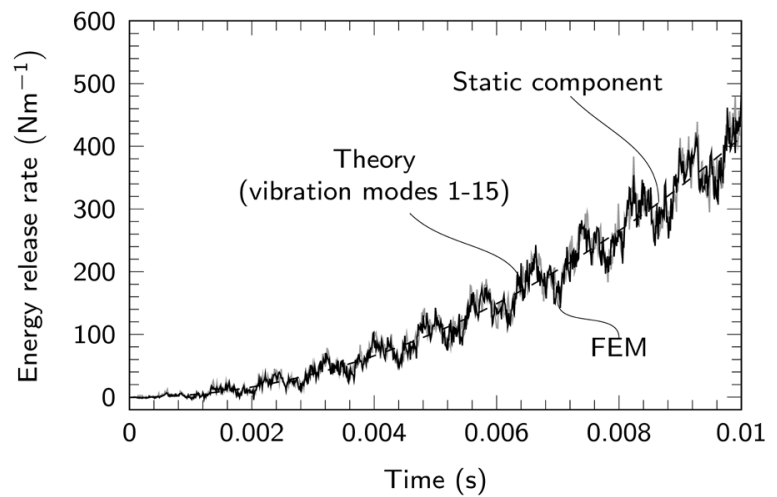

(b)

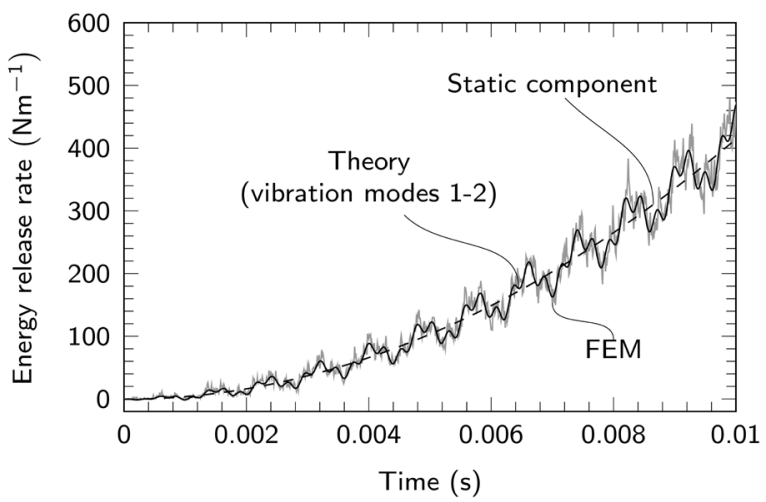

(d)

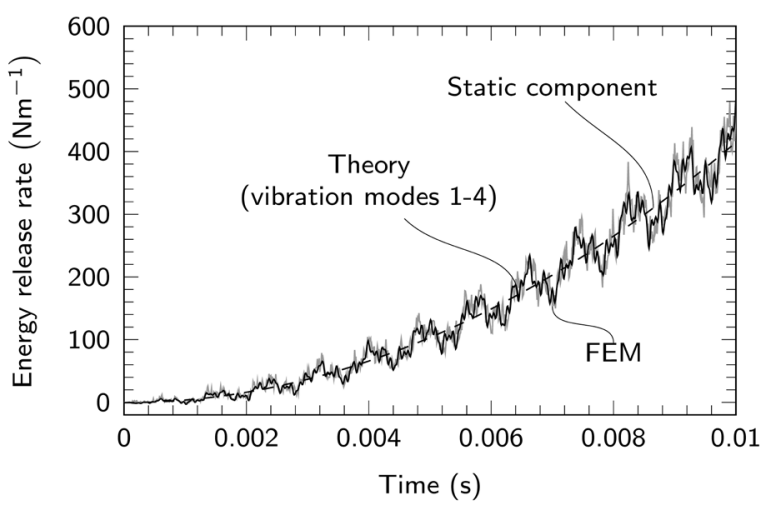

(f)

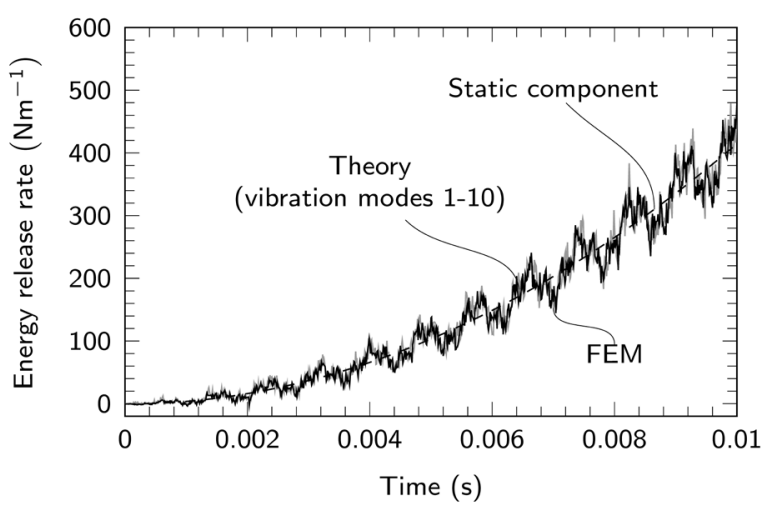

(h)

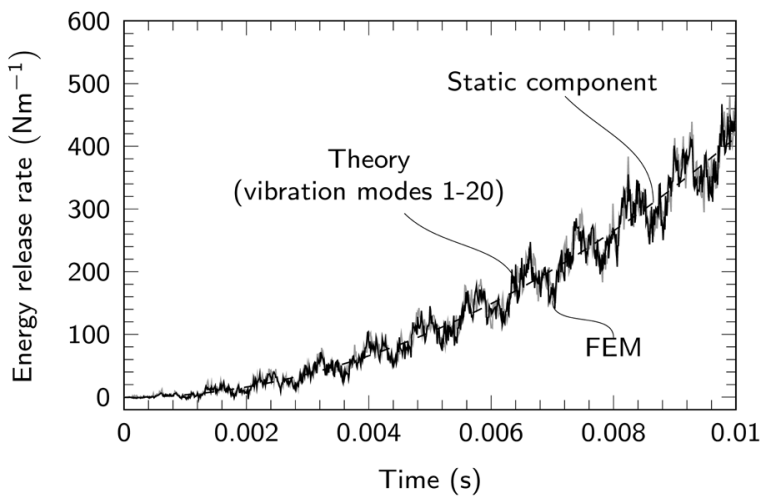

Fig. 7. Dynamic ERR versus time results from developed theory (black line) and from FEM (gray line) with increasing numbers of vibration modes 


\subsection{Non-rigid interfaces}

The same DCB geometry and material properties were used for the second verification study of cracks on non-rigid linear-elastic interfaces. The same FEM model was used except for the uncracked interface ahead of the crack tip being modelled with cohesive elements (COH2D4) of length $0.05 \mathrm{~mm}$ and thickness $0.0001 \mathrm{~mm}$ to simulate the linear-elastic interface with a traction-separation law. A crack closure integral was used at the crack tip to determine the dynamic ERR [23]. Note that the density of the cohesive element was set as $1 \mathrm{~kg} \mathrm{~m}^{-3}$, which is $0.1 \%$ of the DCB material, in order not to add significant extra mass to the system.

Since the DCB in Fig. 6a deforms symmetrically but the developed theory is for a partiallysupported beam on an elastic foundation (Fig. 1), the normal stiffness of the cohesive elements in these FEM simulations must be set as $E_{n n}=0.5 k$. This relationship is a consequence of the symmetric deformation of the DCB (and its interface) under the prescribed loading in comparison to the 'one-sided' deformation of a partially supported beam on an elastic foundation, which the theory is for. As long as the deformation is symmetrical, this relationship between $E_{n n}$ and $k$ is valid.

Four levels of interface stiffness $k$ were examined, namely, 0.1E (1000 MPa), 0.01E $(100 \mathrm{MPa}), 0.001 \mathrm{E}(10 \mathrm{MPa})$ and $0.0001 \mathrm{E}(1 \mathrm{MPa})$, and so the corresponding normal stiffnesses $E_{n n}$ for the cohesive elements were $500 \mathrm{MPa}, 50 \mathrm{MPa}, 5 \mathrm{MPa}$ and $0.5 \mathrm{MPa}$, respectively.

The dynamic ERR versus time results from the FEM and the developed theory (with the first five vibration modes) are compared in Fig. 8, with the black line representing the theory and the gray line representing the FEM. There is excellent agreement between the theory and the FEM for the three different foundation stiffnesses shown in Fig. 8a-c considering the overall magnitude of ERR and the frequencies, phases and amplitudes of oscillation.

Note that the developed theory is only applicable for relatively large foundation stiffness: In Section 2.1.3, the general solution of free vibration of the foundation-supported beam section requires that $4 \alpha_{i}^{4}=k / E I-\beta_{i}^{4}>0$. Furthermore, in Section 2.3.2, the developed expression for dynamic ERR requires that $\gamma L \gtrsim 3$. For $k=0.0001 E$ in Fig. $8 \mathrm{~d}$, the condition of $k / E I-\beta_{i}^{4}>0$ is met only for the first two vibration modes, and then from the third vibration mode onwards, $k / E I-\beta_{i}^{4}<0$. This accounts for the discrepancy seen between the results from the FEM and the developed theory in Fig. 8d. 
(a)

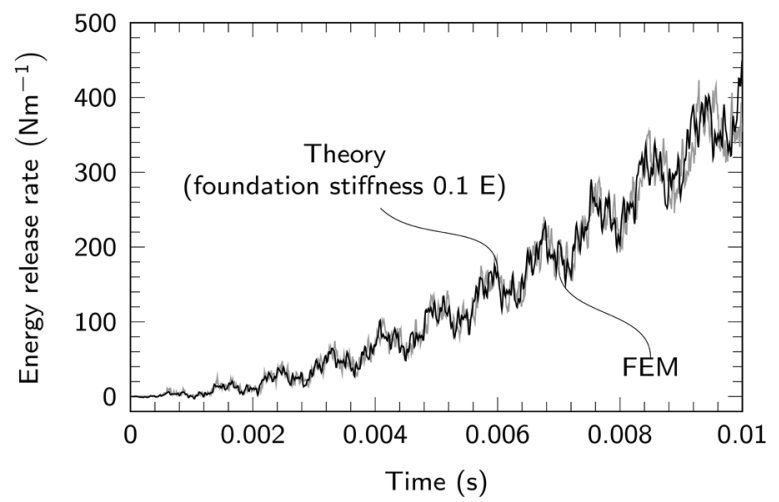

(c)

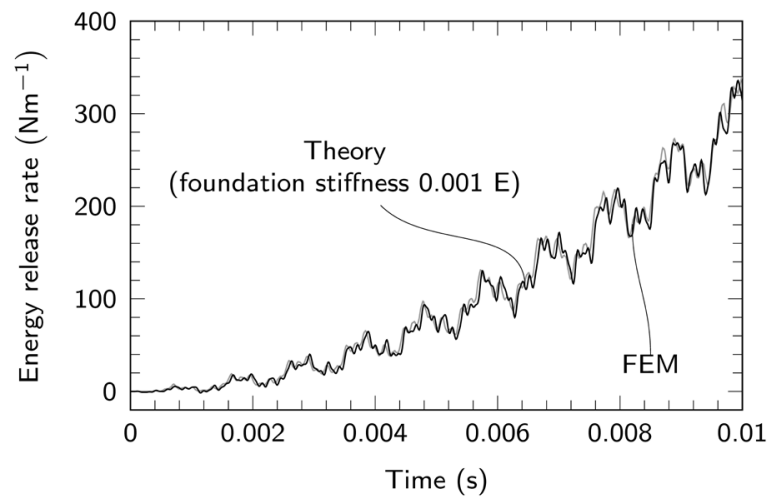

(b)

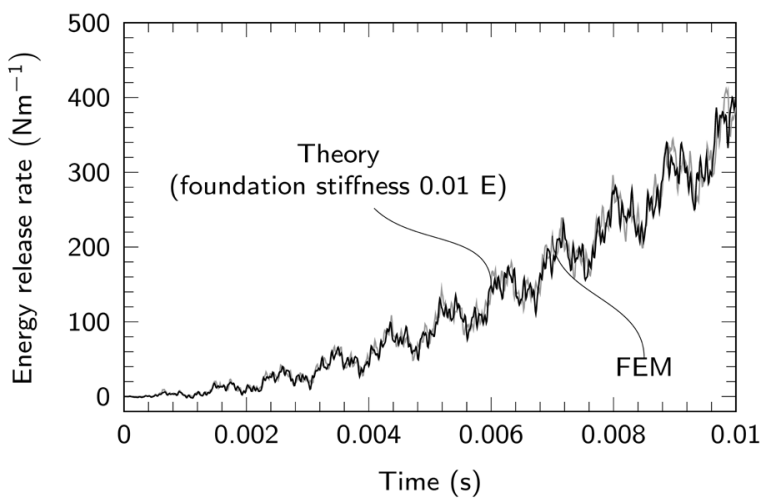

(d)

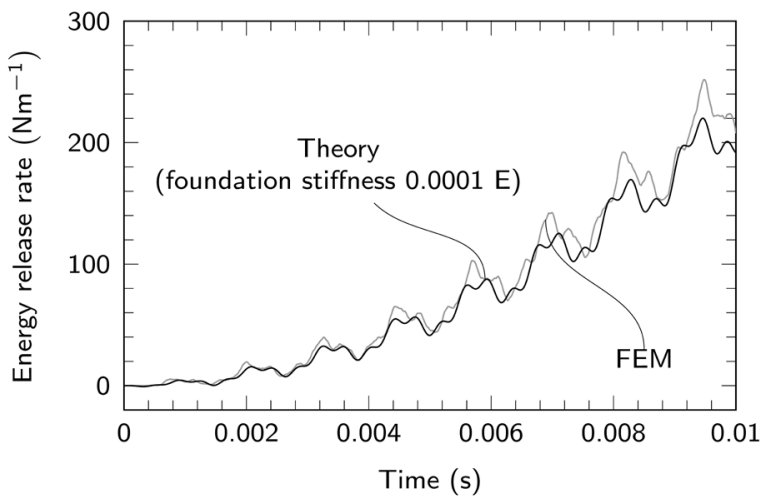

Fig. 8. Dynamic ERR versus time results from developed theory (black line) and from FEM (gray line) with the first five vibration modes for different foundation stiffnesses

\subsection{Fracture mode mixity}

The developed theory also applies to thin layers on thick substrates. It provides the total dynamic ERR; however, due to the asymmetric configuration, the fracture is mixed mode, that is, the total ERR comprises both fracture modes I $\left(G_{\mathrm{I}}\right)$ and II $\left(G_{\mathrm{II}}\right)$. In this third verification study, the agreement between the developed theory and FEM simulations is checked for a crack on a rigid interface between a thin layer and a thick substrate. Furthermore, the fracture mode mixity is calculated and compared against the mixed-mode partition theory in Ref. [24], which was developed for quasi-static fracture of thin layers on thick substrates.

The geometry shown in Fig. $6 \mathrm{~b}$ with a beam width of $1 \mathrm{~mm}$ was used for the third verification study. The same material properties from the first verification study were used, and the same global mesh size of $0.2 \mathrm{~mm}$ was used (without any refinement around the crack tip) giving the total number of elements as 44000. The VCCT was applied to determine the two ERR fracture mode components, $G_{\mathrm{I}}$ and $G_{\mathrm{II}}$, with the total ERR being $G=G_{\mathrm{I}}+G_{\mathrm{II}}$. 
Due to the different geometry, it is expected that a different interface stiffness $k$ (compared to the first verification study) represents a rigid interface. As in the first verification study, a parametric study was conducted using the developed theory to test a range of $k$ values. It was found that using $k=0.5 E$ provides the best agreement with the FEM results.

The total dynamic ERR versus time results from the FEM and the developed theory (with $k=0.5 E$ and the first five vibration modes) are compared in Fig. 8a with the black line representing the theory and the gray line representing the FEM. The total dynamic ERR from the developed theory was then partitioned according to Ref. [24] (which gives $G_{\text {II }} / G_{\text {I }}=0.6059$ ) and is compared against the FEM results in Fig. 8b. Excellent agreement is seen between the developed theory and the FEM for both the total ERR and its partitions, showing that the quasistatic partition theory in Ref. [24] is also applicable under dynamic loading.

(a)

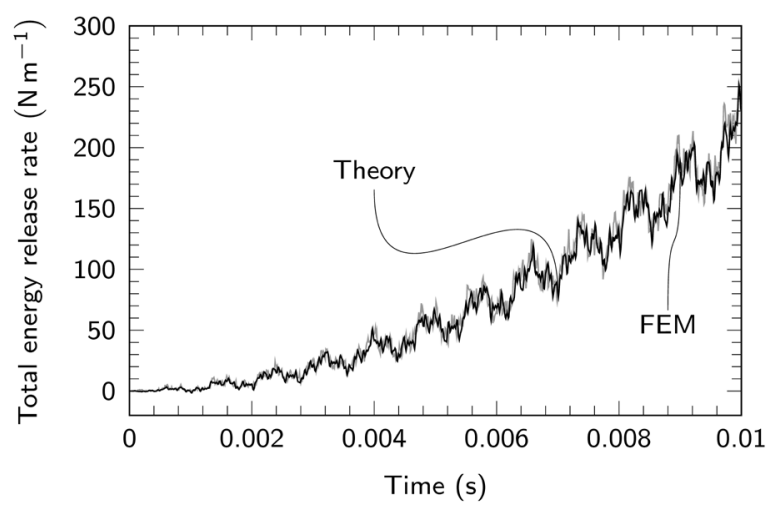

(b)

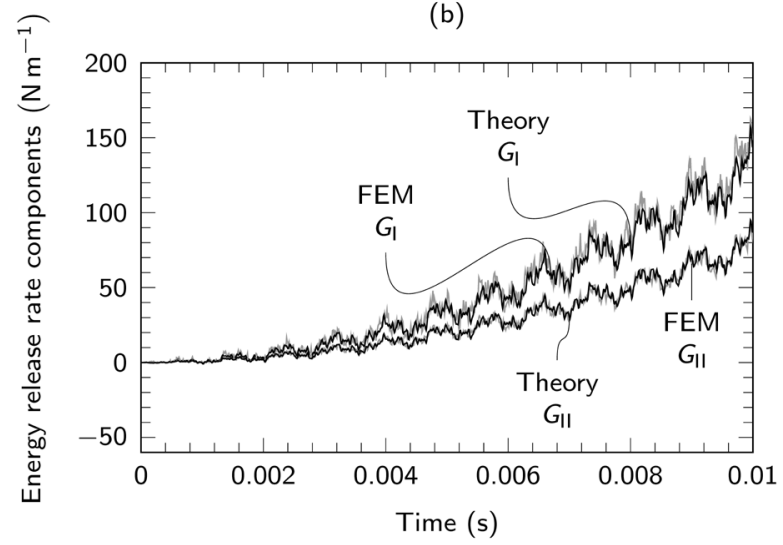

Fig. 9. Results for (a) total dynamic ERR and (b) its fracture-mode I and fracture-mode II components versus time results from developed theory (black line) and from FEM (gray line).

\section{Conclusion}

The dynamic ERR of a stationary delamination on the interface between a partially supported vibrating beam and an elastic foundation with time-dependent displacement applied to the beam's free end was derived analytically. The developed theory is completely analytical and applicable to both symmetric DCBs and thin layers on thick substrates. The dynamic EulerBernoulli beam theory was used to derive the time-dependent deflection, which then permitted the total mechanical energy of the system to be calculated, considering the strain energy of the beam and the elastic foundation, and the kinetic energy of the beam. Finally, by considering energy flux through a contour surrounding the crack tip, and by taking account of the dispersive propagation of flexural waves, the dynamic ERR was derived. It was discovered that the 
dispersive propagation of flexural waves must be considered in order to capture the ERR contributions from higher-order vibration modes. Furthermore, it was found that the elastic interface ahead of the crack tip should be considered in order to correctly capture the phase of ERR oscillation.

A series of verification studies were carried out using the FEM. For rigid interfaces, the interface stiffness in the developed theory needs to be of the same order as the beam material's Young's modulus: for a DCB, an interface stiffness $k=0.35 E$ in the developed theory was found to provide the best agreement; for a thin layer on a thick substrate, it was $k=0.5 E$. With these values of interface stiffness, there is excellent agreement between the theory and the FEM for a rigid interface considering the overall magnitude of ERR and the frequencies, phase and amplitudes of oscillation. Furthermore, in the case of a thin layer on a thick substrate, which gives a mixed-mode fracture, the quasi-static partition theory in Ref. [24] continues to be applicable under dynamic loading to partition the total ERR $G$ into its components, $G_{\text {I }}$ and $G_{\text {II }}$. For non-rigid interfaces, the developed theory is only applicable for relatively large foundation stiffness, that is, the following conditions must be satisfied: $4 \alpha_{i}^{4}=k / E I-\beta_{i}^{4}>0$ and $\gamma L \gtrsim 3$. Under these conditions, there is also excellent agreement between the theory and the FEM considering the overall magnitude of ERR and the frequencies, phases and amplitudes of oscillation.

To the best of the authors' knowledge, the theory of dynamic ERR, which includes higherorder vibration, has not been solved before. The dynamic ERR presented in Eq. (69) is readily applicable to various engineering applications, for example, to determine the dynamic fracture toughness of layered materials in DCB tests, and to characterize the fracture behavior of engineering structures under dynamic loads. Furthermore, the partially supported beam's elastic foundation is particularly relevant for the study of crack process zones, which usually must be studied using the FEM and the cohesive zone model.

Also noteworthy is that current work by the authors, which will soon be reported in a separate publication, shows that the reported theory of dynamic ERR for stationary delamination is also of great value to investigate propagating delamination, also with consideration for vibration. This will be useful to post-process high-loading-rate fracture test data to determine the loading rate-dependent fracture toughness of propagating delamination in laminated or in adhesively bonded structures. 


\section{Appendices}

Appendix A. Boundary conditions and continuity conditions

For the foundation-supported beam section, the boundary conditions for the total deflection $w^{\mathrm{FD}}(x, t)$ are $w^{\mathrm{FD}}(0, t)=0$ and $w^{\mathrm{FD}(1)}(0, t)=0$. By using these boundary conditions for $w^{\mathrm{FD}}(x, t)$ in Eq. (3), and forcing homogeneous conditions, the boundary condition for the freevibration component $w_{\mathrm{fv}}^{\mathrm{FD}}(x, t)$ and the shifting function $F^{\mathrm{FD}}(x)$ are obtained as $w_{\mathrm{fv}}^{\mathrm{FD}}(0, t)=0, w_{\mathrm{fv}}^{\mathrm{FD}(1)}(0, t)=0, F^{\mathrm{FD}}(0)=0$ and $F^{\mathrm{FD}(1)}(0)=0$.

For the free beam section, the boundary conditions for the free-vibration component $w_{\mathrm{fv}}^{\mathrm{FR}}(x, t)$ and the shifting function $F^{\mathrm{FR}}(x)$ are $w_{\mathrm{fv}}^{\mathrm{FR}}(L+a, t)=0, \quad w_{\mathrm{fv}}^{\mathrm{FR}(2)}(L+a, t)=0$, $F^{\mathrm{FR}}(L+a)=1$ and $F^{\mathrm{FR}(2)}(L+a)=0$.

Considering continuity at the crack tip location, $x=L$, since the two beam sections share the same deflection, slope, bending moment and shear force at this location, the following boundary conditions are derived by forcing homogeneous conditions - for the free vibration components: $w_{\mathrm{fv}}^{\mathrm{FD}}(L, t)=w_{\mathrm{fv}}^{\mathrm{FR}}(L, t), \quad w_{\mathrm{fv}}^{\mathrm{FD}(1)}(L, t)=w_{\mathrm{fv}}^{\mathrm{FR}(1)}(L, t), w_{\mathrm{fv}}^{\mathrm{FD}(2)}(L, t)=w_{\mathrm{fv}}^{\mathrm{FR}(2)}(L, t)$ and $w_{\mathrm{fv}}^{\mathrm{FD}(3)}(L, t)=w_{\mathrm{fv}}^{\mathrm{FR}(3)}(L, t)$; and for the shifting functions: $F^{\mathrm{FD}}(L)=F^{\mathrm{FR}}(L)$, $F^{\mathrm{FD}(1)}(L)=F^{\mathrm{FR}(1)}(L), \quad F^{\mathrm{FD}(2)}(L)=F^{\mathrm{FR}(2)}(L)$ and $F^{\mathrm{FD}(3)}(L)=F^{\mathrm{FR}(3)}(L)$.

\section{Appendix B. Derivation of orthogonality condition}

Substituting Eqs. (11) and (12) into Eqs. (7) and (9), and rearranging provides two ordinary differential equations for the normal modes, $W_{i}^{\mathrm{FD}}(x)$ and $W_{i}^{\mathrm{FR}}(x)$, and one ordinary differential equation for the modal displacement $T_{i}(t)$ :

$$
\begin{gathered}
W_{i}^{\mathrm{FD}(4)}(x)+\left(\frac{k}{E I}-\omega_{i}^{2} \frac{\rho A}{E I}\right) W_{i}^{\mathrm{FD}}(x)=0, \\
W_{i}^{\mathrm{FR}(4)}(x)-\omega_{i}^{2} \frac{\rho A}{E I} W_{i}^{\mathrm{FR}}(x)=0, \\
\ddot{T}_{i}(t)+\omega_{i}^{2} T_{i}(t)=0 .
\end{gathered}
$$


The corresponding boundary conditions for the normal modes are $W_{i}^{\mathrm{FD}}(0)=0$, $W_{i}^{\mathrm{FD}(1)}(0)=0, W_{i}^{\mathrm{FR}}(L+a)=0$ and $W_{i}^{\mathrm{FR}(2)}(L+a)=0$, and for the continuity condition at $x=L \quad, \quad W_{i}^{\mathrm{FD}}(L)=W_{i}^{\mathrm{FR}}(L) \quad, \quad W_{i}^{\mathrm{FD}(1)}(L)=W_{i}^{\mathrm{FR}(1)}(L) \quad, \quad W_{i}^{\mathrm{FD}(2)}(L)=W_{i}^{\mathrm{FR}(2)}(L) \quad$ and $W_{i}^{\mathrm{FD}(3)}(L)=W_{i}^{\mathrm{FR}(3)}(L)$.

For the foundation-supported beam section, multiplying Eq. (B1) by $W_{j}^{\mathrm{FD}}(x)$, integrating over the length, and applying the boundary conditions gives

$$
\begin{aligned}
& W_{j}^{\mathrm{FD}}(L) W_{i}^{\mathrm{FD}(3)}(L)-W_{j}^{\mathrm{FD}(1)}(L) W_{i}^{\mathrm{FD}(2)}(L)+\int_{0}^{L} W_{j}^{\mathrm{FD}(2)}(x) W_{i}^{\mathrm{FD}(2)}(x) d x \\
= & -\frac{k}{E I} \int_{0}^{L} W_{i}^{\mathrm{FD}}(x) W_{j}^{\mathrm{FD}}(x) d x+\omega_{i}^{2} \frac{\rho A}{E I} \int_{0}^{L} W_{i}^{\mathrm{FD}}(x) W_{j}^{\mathrm{FD}}(x) d x .
\end{aligned}
$$

For the free beam section, multiplying Eq. (B2) by $W_{j}^{\mathrm{FR}}(x)$, integrating over the length of free section beam (that is, from $L$ to $L+a$ ), and applying the boundary conditions gives

$$
\begin{aligned}
& -W_{j}^{\mathrm{FR}}(L) W_{i}^{\mathrm{FR}(3)}(L)+W_{j}^{\mathrm{FR}(1)}(L) W_{i}^{\mathrm{FR}(2)}(L)+\int_{L}^{L+a} W_{j}^{\mathrm{FR}(2)}(x) W_{i}^{\mathrm{FR}(2)}(x) d x \\
= & \omega_{i}^{2} \frac{\rho A}{E I} \int_{L}^{L+a} W_{i}^{\mathrm{FR}}(x) W_{j}^{\mathrm{FR}}(x) d x .
\end{aligned}
$$

Summing Eqs. (B4) and (B5), applying continuity at the crack tip, and then subtracting this from itself with the subscripts $i$ and $j$ exchanged, gives

$$
\left(\omega_{i}^{2}-\omega_{j}^{2}\right)\left[\int_{0}^{L} \rho A W_{i}^{\mathrm{FD}}(x) W_{j}^{\mathrm{FD}}(x) d x+\int_{L}^{L+a} \rho A W_{i}^{\mathrm{FR}}(x) W_{j}^{\mathrm{FR}}(x) d x\right]=0 .
$$

Since the angular frequency of the system is unique, that is, $\omega_{i} \neq \omega_{j}$ for $i \neq j$, therefore

$$
\int_{0}^{L} \rho A W_{i}^{\mathrm{FD}}(x) W_{j}^{\mathrm{FD}}(x) d x+\int_{L}^{L+a} \rho A W_{i}^{\mathrm{FR}}(x) W_{j}^{\mathrm{FR}}(x) d x=0 .
$$

Now, including the case of $i=j$ and normalizing Eq. (B7), finally the orthogonality of free vibration of a beam partially supported on an elastic foundation is written as

$$
\int_{0}^{L} \rho A W_{i}^{\mathrm{FD}}(x) W_{j}^{\mathrm{FD}}(x) d x+\int_{L}^{L+a} \rho A W_{i}^{\mathrm{FR}}(x) W_{j}^{\mathrm{FR}}(x) d x=\delta_{i j} .
$$

\section{Appendix C. Derivation of initial modal displacement and modal velocity}

The initial value of the $i$ th modal displacement $T_{i}(0)$ can be determined by the following procedure: Substitute Eq. (17) into Eqs. (3) and (4) to obtain the following for foundationsupported beam and free beam sections, respectively: 


$$
\begin{aligned}
& w^{\mathrm{FD}}(x, t)=\sum_{i=1}^{\infty} W_{i}^{\mathrm{FD}}(x)\left[\frac{\dot{T}_{i}(0)}{\omega_{i}} \sin \left(\omega_{i} t\right)+T_{i}(0) \cos \left(\omega_{i} t\right)\right]+F^{\mathrm{FD}}(x) v t, \\
& w^{\mathrm{FR}}(x, t)=\sum_{i=1}^{\infty} W_{i}^{\mathrm{FR}}(x)\left[\frac{\dot{T}_{i}(0)}{\omega_{i}} \sin \left(\omega_{i} t\right)+T_{i}(0) \cos \left(\omega_{i} t\right)\right]+F^{\mathrm{FR}}(x) v t .
\end{aligned}
$$

The initial displacements of these two beam sections are therefore $w^{\mathrm{FD}}(x, 0)=\sum_{i=1}^{\infty} W_{i}^{\mathrm{FD}}(x) T_{i}(0)$ and $w^{\mathrm{FR}}(x, 0)=\sum_{i=1}^{\infty} W_{i}^{\mathrm{FR}}(x) T_{i}(0)$, respectively. Multiply $w^{\mathrm{FD}}(x, 0)$ by $\rho A W_{j}^{\mathrm{FD}}(x)$ and integrate over the foundation-supported beam length (from 0 to $a)$; multiply $w^{\mathrm{FR}}(x, 0)$ by $\rho A W_{j}^{\mathrm{FR}}(x)$ and integrate over the free beam length (from $L$ to $L+a)$; and sum these two integrals to have

$$
\begin{aligned}
& \int_{0}^{L} \rho A W_{j}^{\mathrm{FD}}(x) w^{\mathrm{FD}}(x, 0) d x+\int_{L}^{L+a} \rho A W_{j}^{\mathrm{FR}}(x) w^{\mathrm{FR}}(x, 0) d x \\
& =\int_{0}^{L} \rho A W_{j}^{\mathrm{FD}}(x) \sum_{i=1}^{\infty} W_{i}^{\mathrm{FD}}(x) T_{i}(0) d x+\int_{L}^{L+a} \rho A W_{j}^{\mathrm{FR}}(x) \sum_{i=1}^{\infty} W_{i}^{\mathrm{FR}}(x) T_{i}(0) d x .
\end{aligned}
$$

Finally, by applying the orthogonality of free-vibration condition in Eq. (B8) together with the initial conditions that $w^{\mathrm{FD}}(x, 0)=0$ and $w^{\mathrm{FR}}(x, 0)=0$, the $i$ th modal displacement is found to be zero, that is, $T_{i}(0)=0$. Following a similar procedure, the initial value of the $i$ th modal velocity is found to be

$$
\dot{T}_{i}(0)=-v\left[\int_{0}^{L} \rho A W_{i}^{\mathrm{FD}}(x) F^{\mathrm{FD}}(x) d x+\int_{L}^{L+a} \rho A W_{i}^{\mathrm{FR}}(x) F^{\mathrm{FR}}(x) d x\right] .
$$

\section{Appendix D. Solution of shifting functions}

By applying the four boundary conditions for the continuity condition at the crack tip, at $x=L$, the $P_{1}, P_{2}, P_{3}$ and $P_{4}$ are solved. Combining boundary conditions in Appendix A and the general solutions for $F^{\mathrm{FD}}(x)$ and $F^{\mathrm{FR}}(x)$, the following system of equations is obtained: 


$$
\left[\begin{array}{cccc}
\left(\begin{array}{c}
\cosh (\gamma L) \sin (\gamma L) \\
-\cos (\gamma L) \sinh (\gamma L)
\end{array}\right) & \sinh (\gamma L) \sin (\gamma L) & a^{3} & a \\
2 \gamma \sinh (\gamma L) \sin (\gamma L) & \gamma\left(\begin{array}{l}
\cosh (\gamma L) \sin (\gamma L) \\
+\cos (\gamma L) \sinh (\gamma L)
\end{array}\right) & -3 a^{2} & -1 \\
2 \gamma^{2}\left(\begin{array}{l}
\cosh (\gamma L) \sin (\gamma L) \\
+\cos (\gamma L) \sinh (\gamma L)
\end{array}\right) & 2 \gamma^{2} \cosh (\gamma L) \sin (\gamma L) & 6 a & 0 \\
4 \gamma^{3} \cosh (\gamma L) \cos (\gamma L) & 2 \gamma^{3}\left(\begin{array}{l}
\cos (\gamma L) \sinh (\gamma L) \\
-\cosh (\gamma L) \sin (\gamma L)
\end{array}\right) & -6 & 0
\end{array}\right]=\left(\begin{array}{l}
1 \\
P_{2} \\
P_{3} \\
P_{4}
\end{array}\right)=\left(\begin{array}{l}
0 \\
0 \\
0
\end{array}\right) .
$$

Solving this system of equations gives the coefficients $P_{1}, P_{2}, P_{3}$ and $P_{4}$ as

$$
\begin{aligned}
& P_{1}=\frac{-6[\cosh (\gamma L) \cos (\gamma L)+a \gamma \sinh (\gamma L) \cos (\gamma L)-a \gamma \cosh (\gamma L) \sin (\gamma L)]}{2 a^{3} \gamma^{3}[\cos (2 \gamma L)+\cosh (2 \gamma L)+2]+6 a^{2} \gamma^{2}[\sin (2 \gamma L)+\sinh (2 \gamma L)]+6 a \gamma[\cosh (2 \gamma L)-\cos (2 \gamma L)]+3[\sinh (2 \gamma L)-\sin (2 \gamma L)]}, \\
& P_{2}=\frac{-6[\sinh (\gamma L) \cos (\gamma L)+\cosh (\gamma L) \sin (\gamma L)+2 a \gamma \cosh (\gamma L) \cos (\gamma L)]}{2 a^{3} \gamma^{3}[\cos (2 \gamma L)+\cosh (2 \gamma L)+2]+6 a^{2} \gamma^{2}[\sin (2 \gamma L)+\sinh (2 \gamma L)]+6 a \gamma[\cosh (2 \gamma L)-\cos (2 \gamma L)]+3[\sinh (2 \gamma L)-\sin (2 \gamma L)]}, \\
& P_{3}=\frac{-\gamma^{3}[\cosh (2 \gamma L)+\cos (2 \gamma L)+2]}{2 a^{3} \gamma^{3}[\cos (2 \gamma L)+\cosh (2 \gamma L)+2]+6 a^{2} \gamma^{2}[\sin (2 \gamma L)+\sinh (2 \gamma L)]+6 a \gamma[\cosh (2 \gamma L)-\cos (2 \gamma L)]+3[\sinh (2 \gamma L)-\sin (2 \gamma L)]}, \\
& P_{4}=\frac{3 \gamma\left\{a^{2} \gamma^{2}[\cosh (2 \gamma L)+\cos (2 \gamma L)+2]+2 a \gamma[\sinh (2 \gamma L)+\sin (2 \gamma L)]+[\cosh (2 \gamma L)-\cos (2 \gamma L)]\right\}}{2 a^{3} \gamma^{3}[\cos (2 \gamma L)+\cosh (2 \gamma L)+2]+6 a^{2} \gamma^{2}[\sin (2 \gamma L)+\sinh (2 \gamma L)]+6 a \gamma[\cosh (2 \gamma L)-\cos (2 \gamma L)]+3[\sinh (2 \gamma L)-\sin (2 \gamma L)]} .
\end{aligned}
$$

Appendix E. Derivation of $U_{\mathrm{loc}}=U_{\mathrm{loc} / \mathrm{S}}+U_{\mathrm{loc} / \mathrm{C}}$

The combined results from Sections 2.1.1 to 2.1.3 allow $w_{\mathrm{fv}}^{\mathrm{FD}}(x, t)$ and $w_{\mathrm{fv}}^{\mathrm{FR}}(x, t)$ in Eqs. (22) and (23) to be substituted into Eq. (27) and expanded, giving

$$
U_{\mathrm{loc}}=\frac{1}{2} E I \int_{0}^{L}\left\{-v \sum_{i=1}^{\infty}\left[\frac{H_{i}}{\omega_{i}} W_{i}^{\mathrm{FD}(2)}(x) \sin \left(\omega_{i} t\right)\right]\right\}^{2} d x+\frac{1}{2} E I \int_{L}^{L+a}\left\{-v \sum_{i=1}^{\infty}\left[\frac{H_{i}}{\omega_{i}} W_{i}^{\mathrm{FR}(2)}(x) \sin \left(\omega_{i} t\right)\right]\right\}^{2} d x, \quad(\mathrm{E} 1)
$$

which then further expands as 


$$
\begin{aligned}
& U_{\mathrm{loc}}=\frac{1}{2} E I v^{2} \lim _{n \rightarrow \infty} \int_{0}^{L}\left\{\begin{array}{l}
{\left[\frac{H_{1}}{\omega_{1}} W_{1}^{\mathrm{FD}(2)}(x) \sin \left(\omega_{1} t\right)\right]^{2}+2 \frac{H_{1}}{\omega_{1}} W_{1}^{\mathrm{F}(2)}(x) \sin \left(\omega_{1} t\right) \sum_{j=2}^{n} \frac{H_{j}}{\omega_{j}} W_{j}^{\mathrm{FD}(2)}(x) \sin \left(\omega_{j} t\right)} \\
+\left[\frac{H_{2}}{\omega_{2}} W_{2}^{\mathrm{FD}(2)}(x) \sin \left(\omega_{2} t\right)\right]^{2}+2 \frac{H_{2}}{\omega_{2}} W_{2}^{\mathrm{FD}(2)}(x) \sin \left(\omega_{2} t\right) \sum_{j=3}^{n} \frac{H_{j}}{\omega_{j}} W_{j}^{\mathrm{FD}(2)}(x) \sin \left(\omega_{j} t\right) \\
+\left[\frac{H_{n-1}}{\omega_{n-1}} W_{n-1}^{\mathrm{FD}(2)}(x) \sin \left(\omega_{n-1} t\right)\right]^{2}+2 \frac{H_{n-1}}{\omega_{n-1}} W_{n-1}^{\mathrm{F}(2)}(x) \sin \left(\omega_{n-1} t\right) \frac{H_{n}}{\omega_{n}} W_{n}^{\mathrm{FD}(2)}(x) \sin \left(\omega_{n} t\right) \\
+\left[\frac{H_{n}}{\omega_{n}} W_{n}^{\mathrm{FD}(2)}(x) \sin \left(\omega_{n} t\right)\right]^{2}
\end{array}\right\} d x \\
& +\frac{1}{2} E I v^{2} \lim _{n \rightarrow \infty} \int_{L}^{L+a}\left\{\begin{array}{l}
{\left[\frac{H_{1}}{\omega_{1}} W_{1}^{\mathrm{FR}(2)}(x) \sin \left(\omega_{1} t\right)\right]^{2}+2 \frac{H_{1}}{\omega_{1}} W_{1}^{\mathrm{FR}(2)}(x) \sin \left(\omega_{1} t\right) \sum_{j=2}^{n} \frac{H_{j}}{\omega_{j}} W_{j}^{\mathrm{FR}(2)}(x) \sin \left(\omega_{j} t\right)} \\
+\ldots \\
+\ldots \\
+\left[\frac{H_{2}}{\omega_{2}} W_{2}^{\mathrm{FR}(2)}(x) \sin \left(\omega_{2} t\right)\right]^{2}+2 \frac{H_{2}}{\omega_{2}} W_{2}^{\mathrm{FR}(2)}(x) \sin \left(\omega_{2} t\right) \sum_{j=3}^{n} \frac{H_{j}}{\omega_{j}} W_{j-1}^{\mathrm{FR}(2)}(x) \sin \left(\omega_{j} t\right) \\
+\left[\frac{H_{n}}{\omega_{n}} W_{n}^{\mathrm{FR}(2)}(x) \sin \left(\omega_{n-1} t\right)\right]^{2}+2 \frac{H_{n-1} t}{\omega_{n-1}} W_{n-1}^{\mathrm{FR}(2)}(x) \sin \left(\omega_{n-1} t\right) \frac{H_{n}}{\omega_{n}} W_{n}^{\mathrm{FR}(2)}(x) \sin \left(\omega_{n} t\right)
\end{array}\right\} d x .
\end{aligned}
$$

Eq. (E2) can be rearranged as

$$
\begin{aligned}
& U_{\mathrm{loc}}=\frac{1}{2} E I V^{2} \lim _{n \rightarrow \infty} \int_{0}^{L}\left\{\sum_{i=1}^{n}\left[\frac{H_{i}}{\omega_{i}} W_{i}^{\mathrm{FD}(2)}(x) \sin \left(\omega_{i} t\right)\right]^{2}+2 \sum_{i=1}^{n-1}\left[\frac{H_{i}}{\omega_{i}} W_{i}^{\mathrm{FD}(2)}(x) \sin \left(\omega_{i} t\right) \sum_{j=i+1}^{n} \frac{H_{j}}{\omega_{j}} W_{j}^{\mathrm{FD}(2)}(x) \sin \left(\omega_{j} t\right)\right]\right\} d x \\
& +\frac{1}{2} E I v^{2} \lim _{n \rightarrow \infty} \int_{L}^{L+a}\left\{\sum_{i=1}^{n}\left[\frac{H_{i}}{\omega_{i}} W_{i}^{\mathrm{ER}(2)}(x) \sin \left(\omega_{i} t\right)\right]^{2}+2 \sum_{i=1}^{n-1}\left[\frac{H_{i}}{\omega_{i}} W_{i}^{\mathrm{FR}(2)}(x) \sin \left(\omega_{i} t\right) \sum_{j=i+1}^{n} \frac{H_{j}}{\omega_{j}} W_{j}^{\mathrm{FR}(2)}(x) \sin \left(\omega_{j} t\right)\right]\right\} d x \\
& =\frac{1}{2} E I v^{2} \lim _{n \rightarrow \infty}\left\{\int_{0}^{L} \sum_{i=1}^{n}\left[\frac{H_{i}}{\omega_{i}} W_{i}^{\mathrm{FD}(2)}(x) \sin \left(\omega_{i} t\right)\right]^{2} d x+\int_{L}^{L+a} \sum_{i=1}^{n}\left[\frac{H_{i}}{\omega_{i}} W_{i}^{\mathrm{FR}(2)}(x) \sin \left(\omega_{i} t\right)\right]^{2} d x\right\} \\
& +E I v^{2} \lim _{n \rightarrow \infty}\left\{\begin{array}{l}
\int_{0}^{L} \sum_{i=1}^{n-1}\left[\frac{H_{i}}{\omega_{i}} W_{i}^{\mathrm{F}(2)}(x) \sin \left(\omega_{i} t\right) \sum_{j=i+1}^{n} \frac{H_{j}}{\omega_{j}} W_{j}^{\mathrm{F}(2)}(x) \sin \left(\omega_{j} t\right)\right] d x \\
+\int_{L}^{L+a} \sum_{i=1}^{n-1}\left[\frac{H_{i}}{\omega_{i}} W_{i}^{\mathrm{FR}(2)}(x) \sin \left(\omega_{i} t\right) \sum_{j=i+1}^{n} \frac{H_{j}}{\omega_{j}} W_{j}^{\mathrm{FR}(2)}(x) \sin \left(\omega_{j} t\right)\right]
\end{array}\right\} \\
& =\frac{1}{2} E I v^{2} \sum_{i=1}^{\infty} \frac{H_{i}^{2}}{\omega_{i}^{2}} \sin ^{2} \omega_{i} t\left\{\int_{0}^{L}\left[W_{i}^{\mathrm{FD}(2)}(x)\right]^{2} d x+\int_{L}^{L+a}\left[W_{i}^{\mathrm{FR}(2)}(x)\right]^{2} d x\right\} \\
& +E I v^{2} \lim _{n \rightarrow \infty}\left\{\begin{array}{l}
\int_{0}^{L} \sum_{i=1}^{n-1}\left[\frac{H_{i}}{\omega_{i}} W_{i}^{\mathrm{FD}(2)}(x) \sin \left(\omega_{i} t\right) \sum_{j=i+1}^{n} \frac{H_{j}}{\omega_{j}} W_{j}^{\mathrm{FR}(2)}(x) \sin \left(\omega_{j} t\right)\right] d x \\
+\int_{L}^{L+a} \sum_{i=1}^{n-1}\left[\frac{H_{i}}{\omega_{i}} W_{i}^{\mathrm{FR}(2)}(x) \sin \left(\omega_{i} t\right) \sum_{j=i+1}^{n} \frac{H_{j}}{\omega_{j}} W_{j}^{\mathrm{FR}(2)}(x) \sin \left(\omega_{j} t\right)\right]
\end{array}\right\} .
\end{aligned}
$$

The first and second terms of Eq. (E3) are $U_{\text {loc/S }}$ and $U_{\text {loc/C }}$ in Eqs. (29) and (30), respectively.

Appendix F. Simplification of $U_{\mathrm{loc} / \mathrm{S}}$ and $U_{\mathrm{loc} / \mathrm{C}}$

Summing Eqs. (B4) and (B5) and applying continuity conditions at the crack tip gives 


$$
\begin{aligned}
& \int_{0}^{L} W_{j}^{\mathrm{FD}(2)}(x) W_{i}^{\mathrm{FD}(2)}(x) d x+\int_{L}^{L+a} W_{j}^{\mathrm{FR}(2)}(x) W_{i}^{\mathrm{FR}(2)}(x) d x \\
& =-\frac{k}{E I} \int_{0}^{L} W_{i}^{\mathrm{FD}}(x) W_{j}^{\mathrm{FD}}(x) d x \\
& +\omega_{i}^{2} \frac{\rho A}{E I}\left[\int_{0}^{L} W_{i}^{\mathrm{FD}}(x) W_{j}^{\mathrm{FD}}(x) d x+\int_{L}^{L+a} W_{i}^{\mathrm{FR}}(x) W_{j}^{\mathrm{FR}}(x) d x\right] .
\end{aligned}
$$

Then by letting $i=j$, and using the orthogonality condition in Eq. (B8), Eq. (F1) simplifies to

$$
\int_{0}^{L}\left[W_{i}^{\mathrm{FD}(2)}(x)\right]^{2} d x+\int_{L}^{L+a}\left[W_{i}^{\mathrm{FR}(2)}(x)\right]^{2} d x=-\frac{k}{E I} \int_{0}^{L}\left[W_{i}^{\mathrm{FD}}(x)\right]^{2} d x+\frac{\omega_{i}^{2}}{E I}
$$

Now $U_{\text {loc/s }}$ in Eq. (29) can be simplified to Eq. (48) by using Eq. (F2); and $U_{\text {loc/C }}$ in Eq. (30) can be simplified to Eq. (49).

Appendix G. Derivation of $U_{\mathrm{cp}}=-S_{\mathrm{cp}}$

Expanding $U_{\mathrm{cp}}^{\mathrm{FD}}$, which is the first term of Eq. (31), with Eq. (11) and integrating twice by parts gives

$$
U_{\mathrm{cp}}^{\mathrm{FD}}=-E I v^{2} t \sum_{i=1}^{\infty} \frac{H_{i}}{\omega_{i}} \sin \left(\omega_{i} t\right)\left\{\begin{array}{l}
{\left.\left[W_{i}^{\mathrm{FD}(1)}(x) F^{\mathrm{FD}(2)}(x)\right]\right|_{0} ^{L}} \\
-\left.\left[W_{i}^{\mathrm{FD}}(x) F^{\mathrm{FD}(3)}(x)\right]\right|_{0} ^{L} \\
+\int_{0}^{L}\left[W_{i}^{\mathrm{FD}}(x) F^{\mathrm{FD}(4)}(x)\right] d x
\end{array}\right\} .
$$

Then, by using the boundary conditions for $w_{\mathrm{fv}}^{\mathrm{FD}}(x, t)$ and $F^{\mathrm{FD}}(x)$ in Appendix $\mathrm{A}$ and substituting in Eq. (8), $U_{\mathrm{cp}}^{\mathrm{FD}}$ simplifies to

$$
U_{\mathrm{cp}}^{\mathrm{FD}}=-E I v^{2} t \sum_{i=1}^{\infty} \frac{H_{i}}{\omega_{i}} \sin \left(\omega_{i} t\right)\left\{\begin{array}{l}
{\left[W_{i}^{\mathrm{FD}(1)}(L) F^{\mathrm{FD}(2)}(L)\right]} \\
-\left[W_{i}^{\mathrm{FD}}(L) F^{\mathrm{FD}(3)}(L)\right] \\
-\frac{k}{E I} \int_{0}^{L}\left[W_{i}^{\mathrm{FD}}(x, t) F^{\mathrm{FD}}(x)\right] d x
\end{array}\right\} .
$$

By using the same procedure with Eqs. (10) and (12), $U_{\mathrm{cp}}^{\mathrm{FR}}$, which is the second term in Eq. (31), simplifies to

$$
U_{\mathrm{cp}}^{\mathrm{FR}}=-E I v^{2} t \sum_{i=1}^{\infty} \frac{H_{i}}{\omega_{i}} \sin \left(\omega_{i} t\right)\left\{\begin{array}{l}
-W_{i}^{\mathrm{FR}(1)}(L) F^{\mathrm{FR}(2)}(L) \\
+W_{i}^{\mathrm{FR}}(L) F^{\mathrm{FR}(3)}(L)
\end{array}\right\} .
$$


Now $U_{\text {cp }}$ in Eq. (50) is the sum of Eqs. (G2) and (G3) with continuity conditions at the crack tip applied as per Appendix A.

\section{Appendix H. Simplification of $U_{\text {st }}$}

Partially integrating $U_{\mathrm{st}}^{\mathrm{FD}}$ in Eq. (32) twice, and using the boundary conditions described in Appendix A together with Eq. (8), gives

$$
U_{\mathrm{st}}^{\mathrm{FD}}=\frac{1}{2} E I v^{2} t^{2}\left\{F^{\mathrm{FD}(1)}(L) F^{\mathrm{FD}(2)}(L)-F^{\mathrm{FD}(3)}(L) F^{\mathrm{FD}}(L)-\frac{k}{E I} \int_{0}^{L}\left[F^{\mathrm{FD}}(x)\right]^{2} d x\right\} .
$$

By the same procedure for $U_{\mathrm{st}}^{\mathrm{FR}}$ in Eq. (32), but this time using Eq. (10),

$$
U_{\mathrm{st}}^{\mathrm{FR}}=\frac{1}{2} E I v^{2} t^{2}\left\{-F^{\mathrm{FR}(1)}(L) F^{\mathrm{FR}(2)}(L)+F^{\mathrm{FR}(3)}(L) F^{\mathrm{FR}}(L)-F^{\mathrm{FR}(3)}(L+a)\right\} .
$$

Now $U_{\text {st }}$ in Eq. (51) is the sum of Eqs. (H1) and (H2) with continuity conditions at the crack tip applied as per Appendix A together with Eq. (47).

\section{Appendix I. Derivation of $d \omega_{i} / d a$}

Let $D_{i}$ represent the determinant of coefficient matrix in Eq. (15). It is a function of the wavenumbers, $\alpha_{i}$ and $\beta_{i}$, and lengths of the two beam sections, $a$ and $L$. For Eq. (15) to have non-zero solutions, $D_{i}=0$. The total derivative of $D_{i}$ is therefore

$$
d D_{i}\left(\alpha_{i}, \beta_{i}, L, a\right)=\frac{\partial D_{i}}{\partial \alpha_{i}} d \alpha_{i}+\frac{\partial D_{i}}{\partial \beta_{i}} d \beta_{i}+\frac{\partial D_{i}}{\partial L} d L+\frac{\partial D_{i}}{\partial a} d a=0 .
$$

Dividing Eq. (I1) by $d a$ gives

$$
\frac{\partial D_{i}}{\partial \alpha_{i}} \frac{d \alpha_{i}}{d a}+\frac{\partial D_{i}}{\partial \beta_{i}} \frac{d \beta_{i}}{d a}+\frac{\partial D_{i}}{\partial L} \frac{d L}{d a}+\frac{\partial D_{i}}{\partial a}=0
$$

Note that $L+a=$ constant, which gives $d L / d a=-1$; and that $4 \alpha_{i}^{4}=k / E I-\beta_{i}^{4}$ (Section 2.1), which gives $d \alpha_{i} / d a=-\beta_{i}^{3} /\left(4 \alpha_{i}^{3}\right)\left(d \beta_{i} / d a\right)$. By also using $\beta_{i}^{4}=\omega_{i}^{2} \rho A /(E I)$ (Section 2.1), $d \omega_{i} / d a$ is obtained as

$$
\frac{d \omega_{i}}{d a}=2 \beta_{i} \sqrt{\frac{E I}{\rho A}} \frac{d \beta_{i}}{d a}=2 \beta_{i} \frac{\frac{\partial D_{i}}{\partial L}-\frac{\partial D_{i}}{\partial a}}{-\frac{\partial D_{i}}{\partial \alpha_{i}} \frac{\beta_{i}^{3}}{4 \alpha_{i}^{3}}+\frac{\partial D_{i}}{\partial \beta_{i}}} \sqrt{\frac{E I}{\rho A}} .
$$


Appendix J. Normalized dynamic factor due to vibration of various foundation stiffness

Table J.1. Normalized dynamic factors due to vibration with various foundation stiffnesses

\begin{tabular}{cccc}
\hline Foundation stiffness & Vibration mode 1 & Vibration mode 2 & Vibration mode 3 \\
\hline$k / E=1$ & 1 & 0.65599 & 0.48112 \\
$k / E=0.1$ & 1.17225 & 0.67009 & 0.45369 \\
$k / E=0.01$ & 1.19499 & 0.70870 & 0.52466 \\
$k / E=0.001$ & 1.27728 & 0.70111 & 0.49133 \\
\hline
\end{tabular}

\section{Data availability}

The authors confirm that the data supporting the findings of this study are available within the article.

\section{References}

[1] M. Colin de Verdiere, A. A. Skordos, M. May, and A. C. Walton, "Influence of loading rate on the delamination response of untufted and tufted carbon epoxy non crimp fabric composites: Mode I,” Eng. Fract. Mech., vol. 96, pp. 11-25, 2012.

[2] M. Isakov, M. May, P. Hahn, H. Paul, and M. Nishi, "Fracture toughness measurement without force data - Application to high rate DCB on CFRP," Compos. Part A Appl. Sci. Manuf., vol. 119, pp. 176-187, 2019.

[3] A. J. Smiley and R. B. Pipes, "Rate effects on mode I interlaminar fracture toughness in composite materials," J. Compos. Mater., vol. 21, no. 7, pp. 670-687, 1987.

[4] B. R. K. Blackman, A. J. Kinloch, Y. Wang, and J. G. Williams, "The failure of fibre composites and adhesively bonded fibre composites under high rates of test," J. Mater. Sci., vol. 31, no. 17, pp. 4451-4466, 1996.

[5] H. Liu, H. Nie, C. Zhang, and Y. Li, "Loading rate dependency of Mode I interlaminar fracture toughness for unidirectional composite laminates," Compos. Sci. Technol., vol. 167, pp. 215-223, 2018.

[6] R. A. Cidade et al., "Determination of mode I dynamic fracture toughness of IM78552 composites by digital image correlation and machine learning," Compos. Struct., vol. 210, pp. 707-714, 2019.

[7] R. W. Bedsole, P. B. Bogert, and H. V. Tippur, "An experimental investigation of interlaminar and intralaminar dynamic fracture of CFRPs: Effect of matrix 
modification using carbon nanotubes," Compos. Struct., vol. 132, pp. 1043-1055, 2015.

[8] Y. Liu, F. P. van der Meer, and L. J. Sluys, "Cohesive zone and interfacial thick level set modeling of the dynamic double cantilever beam test of composite laminate," Theor. Appl. Fract. Mech., vol. 96, pp. 617-630, 2018.

[9] Z. Kang, T. Q. Bui, D. D. Nguyen, and S. Hirose, "Dynamic stationary crack analysis of isotropic solids and anisotropic composites by enhanced local enriched consecutiveinterpolation elements," Compos. Struct., vol. 180, pp. 221-233, 2017.

[10] K. Huang, L. Guo, and H. Yu, "Investigation on mixed-mode dynamic stress intensity factors of an interface crack in bi-materials with an inclusion," Compos. Struct., vol. 202, pp. 491-499, 2018.

[11] T. Chen, C. M. Harvey, S. Wang, and V. V. Silberschmidt, "Dynamic interfacial fracture of a thin-layered structure," Procedia Struct. Integr., vol. 13, pp. 613-618, 2018 .

[12] T. Chen, C. M. Harvey, S. Wang, and V. V. Silberschmidt, "Dynamic interfacial fracture of a double cantilever beam," Engineering Fracture Mechanics, 2018.

[13] D. A. Grant, "Beam vibrations with time-dependent boundary conditions," J. Sound Vib., vol. 89, no. 4, pp. 519-522, 1983.

[14] S. S. Rao, Vibration of Continuous Systems. John Wiley \& Sons, 2007.

[15] L.B.Freund, Dynamic fracture mechanics. Cambridge University Press, 1990.

[16] K.F.Graff, Wave Motion in Elastic Solids. Oxford University Press, 1991.

[17] S.Gopalakrishnan, Wave Propagation in Materials and Structures. CRC Press, 2017.

[18] S. Wang, C. M. Harvey, and L. Guan, "Partition of mixed modes in layered isotropic double cantilever beams with non-rigid cohesive interfaces," Eng. Fract. Mech., vol. 111, pp. 1-25, 2013.

[19] M. F. Kanninen, “An augmented double cantilever beam model for studying crack propagation and arrest," Int. J. Fract., vol. 9, no. 1, pp. 83-92, 1973.

[20] A. P. Gallagher, "Bending of a Free Beam on an Elastic Foundation," J. Appl. Mech., vol. 50, no. 2, p. 463, 2009.

[21] A. B. Vesic, "Beams on Elastic Subgrade and the Winkler's Hypothesis," Proc. 5th Int. Conf. Soil Mech., pp. 845-850, 1963.

[22] A. Turon, C. G. Dávila, P. P. Camanho, and J. Costa, "An engineering solution for mesh size effects in the simulation of delamination using cohesive zone models," Eng. Fract. Mech., vol. 74, no. 10, pp. 1665-1682, 2007. 
[23] C. J. Jih and C. T. Sun, "Evaluation of a finite element based crack-closure method for calculating static and dynamic strain energy release rates," Eng. Fract. Mech., vol. 37, no. 2, pp. 313-322, 1990.

[24] J. D. Wood, C. M. Harvey, and S. Wang, "Adhesion toughness of multilayer graphene films," Nat. Commun., vol. 8, no. 1, 2017. 